Ann. Biol. anim. Bioch. Biophys., I969, 9 (4), 6от-625.

\title{
INFLUENCE DE L'APPORT DE VITAMINES B SUR LA CONSOMMATION SPONTANEE DE PRINCIPES ÉNERGÉTIQUES CHEZ LE RAT BLANC EN CROISSANCE
}

\author{
Y. HENRY et A. RÉRAT, \\ avec la collaboration technique de Janine Jung, \\ Yvonne Dantel, Jacqueline Lecterc et M. Lecourtier. \\ Station de Recherches sur l'Élevage des Porcs, \\ Centre national de Recherches zootechniques, 78-Jouy-en-Josas, \\ Institut national de la Recherche agronomique
}

SOMMAIRE

Trois expériences (A, B et $\mathrm{C}$ ) ont été réalisées sur $\mathrm{I} 55$ rats mâles de souche Wistar $\mathrm{CF}$, après sevrage, afin d'étudier les effets d'une polycarence plus ou moins sévère en vitamines $B$ (exp. A) ou de carences simples en thiamine (exp. B) et en riboflavine (exp. C) sur la consommation spontanée d'énergie, les performances de croissance et l'utilisation métabolique des nutriments azotés et énergétiques. Après avoir été préalablement carencés en vitamines, les animaux sont soumis à la technique des repas séparés. A cet effet, ils reçoivent, dans deux mangeoires :

- un régime protidique, apportant une quantité fixe journalière de protéines, sous forme de caséine dévitaminée, additionnée de cystine $(\mathrm{I}, 2 \mathrm{~g}$ dans l'expérience $\mathrm{A}$ et $\mathrm{I}, 8 \mathrm{~g}$ dans les expériences $B$ et $C$ ), et trois niveaux différents de vitamines : une dose normale, correspondant aux recommandations habituelles, une dose réduite au I/Io ou au I/20 de la précédente,

- un régime protéiprive à base de saccharose, alloué ad libitum.

A l'issue des expériences, qui ont duré, soit 28 jours (exp. A et C), soit $\mathrm{I}_{4}$ jours (exp. B), il a été procédé à l'analyse chimique des carcasses, après prélèvement de l'intestin et de son contenu (expérience A) et du foie (dans les trois expériences), ainsi qu'aux dosages de thiamine et de riboflavine dans ces trois fractions, par voie microbiologique.

$L$ 'induction $d$ 'une carence plus ou moins intense en vitamines $B$ se traduit par un ralentissement de la croissance, d'autant plus prononcé que la carence est plus forte. Cette dépression de croissance est accompagnée d'une diminution de la consommation spontanée de principes énergétiques, mais cette dernière (EI) est dans tous les cas directement proportionnelle à la quantité d'azote (N) fixée dans les tissus, suivant l'équation :

$$
\mathrm{E}_{\mathrm{I}}(\mathrm{kcal} / \mathrm{j})=18,74+0,309 \mathrm{~N}(\mathrm{mg} / \mathrm{j}) \text {, }
$$

le coefficient de corrélation étant $\mathrm{r}=0,976$.

L'évolution des teneurs en vitamines dans l'intestin, le foie et la carcasse fait apparaître, principalement dans le foie, une relation étroite entre le dépôt de thiamine dans les tissus et le niveau d'ingestion de cette vitamine, alors que le dépôt de riboflavine s'accroît proportionnellement au gain de poids, et plus précisément à la rétention azotée.

Les résultats obtenus permettent d'exclure une action directe des vitamines sur l'appétit. Ces dernières agiraient seulement indirectement en réglant le métabolisme, selon des modalités particulières à chacune des vitamines. Il s'agit là d'un phénomène général d'ajustement de la consommation d'énergie en fonction du niveau du facteur limitant de la protéinogenèse. 


\section{INTRODUCTION}

La mise en œuvre de la technique des repas séparés, chez le jeune rat, nous a permis de montrer que, lorsque ce dernier reçoit une quantité limitée de matières azotées et des substances énergétiques à volonté, il ajuste la consommation de ces dernières en fonction de la vitesse de croissance permise par l'apport azoté, quantitatif ou qualitatif, et plus précisément en fonction de l'intensité de la protéinogènèse (HENRY et RÉRAT, I962; RÉRAT, HENRY et JACQUOT, I963; RÉRAT et HENRY, I963; HENRY et RÉRAT, I966). Dans ces conditions, l'évolution des dépôts azotés et lipidiques dans les tissus se traduit par une composition corporelle constante, à un poids donné, quelle que soit la nature de la source azotée. On peut se demander si ce phénomène d'ajustement de la consommation d'énergie en fonction de l'intensité de la protéinogènèse ne constitue pas une loi générale qui se retrouve en particulier lorsqu'on fait varier la nature des facteurs pouvant modifier la protéinogènèse ellemême, par exemple le niveau d'ingestion des vitamines ou des minéraux.

Afin de vérifier la validité de cette hypothèse, nous avons entrepris d'étudier, au cours d'une série de trois expériences sur des rats mâles en croissance de souche Wistar $C F$, les effets d'une polycarence en vitamines $\mathrm{B}$ et de carences simples en certaines de ces vitamines (thiamine, riboflavine) sur le mécanisme de régulation à long terme de l'appétit. Certains de ces résultats ont déjà été rapportés par ailleurs (HENRY et RÉRAT, I964; RÉRAT et HENRY, Ig66).

\section{MATÉRIEL ET MÉTHODES}

\section{Expérience $A$ : Influence d'une polycarence en vitamines $B$}

Trente-cinq rats au sevrage, pesant entre 40 et $60 \mathrm{~g}$, sont nourris d'un régime totalement dépourvu de vitamines $B$ et renfermant $18 \mathrm{p}$. Ioo de caséine dévitaminée, additionnée de cystine. Cette privation de vitamines $B$ se prolonge pendant $\mathrm{I} 7$ jours jusqu'à l'obtention d'un arrêt de croissance et même d'une légère perte de poids pendant 4 jours. A l'issue de cette période, 3 lots de 9 animaux sont constitués suivant la méthode des blocs, d'après le poids et le gain de poids, tandis qu'un lot témoin de 6 animaux est sacrifié immédiatement après un jeûne de 8 heures. A ces trois lots, on administre chaque jour, simultanément et dans deux mangeoires séparées :

- une quantité limitée d'un régime protidique $(3,3 \mathrm{~g} / \mathrm{j})$ apportant $\mathbf{I}, 2 \mathrm{~g}$ de protéines, soit 200 mg d'azote environ, sous forme de caséine dévitaminée additionnée de cystine et 3 niveaux de vitamines B : un apport normal, correspondant aux doses habituellement recommandées (lot IA); un apport réduit au I/ io du précédent (lot II A) et au I/20 (lot III A);

- un régime protéiprive à base de saccharose, alloué à volonté.

Les quantités quotidiennes de vitamines, considérées comme normales (lot I A), ont été calculées pour couvrir largement les normes habituellement recommandées : Chlorhydrate de thiamine, $53 \mu \mathrm{g}$; acide nicotinique, $200 \mu \mathrm{g}$; Pyridoxine, $53 \mu \mathrm{g}$; Riboflavine $100 \mu \mathrm{g}$; pantothénate de calcium, $200 \mu \mathrm{g}$; vitamine $\mathrm{B}_{12}, 0,2 \mu \mathrm{g}$; acide folique, $6,6 \mu \mathrm{g}$; Inositol, $3.3 \mathrm{mg}$; acide paraaminobenzoïque, $3,3 \mathrm{mg}$; Biotine, o,or $\mu \mathrm{g}$; Choline, $6,7 \mathrm{mg}$.

Tous les animaux reçoivent en outre $200 \mathrm{mg}$ de vitamine $E$ et $33 \mathrm{mg}$ de vitamine $K$ par jour. Les vitamines $A$ et $D$ sont introduites dans les régimes protidique et protéiprive aux doses respectives de 16 et 3,2 UI par $\mathrm{g}$.

Les régimes, dont la composition est décrite dans le tableau I $\mathrm{A}$, sont distribués à l'état humide, les proportions respectives d'aliment sec et d'eau étant $\mathbf{I}: 0,8$ pour le régime protidique et $\mathrm{I}: \mathrm{o}, \mathrm{I} 5$ pour le régime protéiprive. 
La durée de l'expérience est de 4 semaines. Les animaux sont élevés en cages individuelles à la température de $23^{\circ} \mathrm{C}$. Ils sont pesés deux fois par semaine et les consommations des deux régimes, protidique et protéiprive, sont enregistrées quotidiennement. A la fin de l'expérience, les rats sont sacrifiés au gaz d'éclairage, après un jen̂ne préalable de 8 heures. On isole de la carcasse le foie et la totalité de l'intestin avec son contenu. Sur ces trois fractions, on procède à l'analyse chimique globale :

- matière sèche, par séchage à l'étuve à $95^{\circ} \mathrm{C}$ pendant 24 heures ;

- cendres, par incinération à $55^{\circ} \mathrm{C}$ pendant 36 heures ;

- azote, par la méthode macro KJELDAHL ;

- les lipides sont estimés par différence :

$$
\text { poids frais }- \text { [eau }+ \text { cendres }+ \text { protéines }(\mathrm{N} \times 6,25)] \text {. }
$$

TABLEAU I A

Composition des régimes

(Expérience A)

\begin{tabular}{|c|c|c|c|}
\hline & \multirow{2}{*}{$\begin{array}{c}\text { Régime } \\
\text { préexpérimental }\end{array}$} & \multicolumn{2}{|c|}{ Régime expérimental } \\
\hline & & protidique & protéiprive \\
\hline \multicolumn{4}{|l|}{ Composition p. 100} \\
\hline Caséine dévitaminée Byla extraite 3 fois ..... & 18 & 43,5 & \\
\hline L. Cystine $\ldots \ldots \ldots \ldots \ldots \ldots \ldots \ldots \ldots$ & 0,3 & 1,1 & \\
\hline Sucre cristallisé $\ldots \ldots \ldots \ldots \ldots \ldots \ldots$ & 68,7 & 40,4 & 87 \\
\hline Huile d'arachide ( + vitamines $A$ et $D$ ) ( ${ }^{(1)} \ldots$. & 8 & 8 & 8 \\
\hline Cellulose de bois $\ldots \ldots \ldots \ldots \ldots \ldots \ldots$ & 2 & 2 & 2 \\
\hline Mélange salin (OSBORne et MEndeL)..... & 3 & 3 & 3 \\
\hline Mélange vitaminique $\left({ }^{2}\right) \ldots \ldots \ldots \ldots \ldots \ldots$ & - & 2 & - \\
\hline Résultats moyens d'analyse & & & \\
\hline N p. 100 matière sèche $\ldots \ldots \ldots \ldots \ldots \ldots$ & & 6,52 & \\
\hline Énergie, cal/g matière sèche $\ldots \ldots \ldots \ldots \ldots$ & & 5115 & 4032 \\
\hline
\end{tabular}

(1) A raison de 16 UI de vitamine $A$ et 3,2 UI de vitamine $D_{2}$ par g de régime.

(2) Mélange apportant les quantités suivantes en $\mu \mathrm{g}$ par $\mathrm{g}$ de régime, respectivement pour les lots $\mathrm{A}$, I3 et C ; Chlorhydrate de thiamine, 16-1,6-0,08; Acide nicotinique, 60-6,0-3,0; pyridoxine, 16-1,6-0,08; Riboflavine, 30-3,0-1,5 ; Pantothénate de $\mathrm{Ca}, 60-6,0-3,0$; Vitamine $\mathrm{B}_{12}, 0,06-0,006-0,003$; Acide folique, 2,0-0,2-0,1; Inositol, 1 000-100-50; Acide paraaminobenzoïque, $1000-100-50$; Vitamine E, 60-60-60; Vitamine $K$, 10-10-10. La biotine et la choline sont introduites, sous forme de solution, aux doses respectives de 0,4 $\mu$ g et $2 \mathrm{mg}$ (lot A), 0,04 $\mu$ g et $0,2 \mathrm{mg}$ (Lot B) ; 0,02 et 0,1 $\mathrm{mg}$ (Lot C).

La valeur énergétique des régimes est déterminée à l'aide d'un calorimètre adiabatique Gallenkamp, tandis que le contenu énergétique des carcasses est déduit des teneurs en protéines et en lipides, en utilisant les coefficients d'ATwater et BRYANT (1903) (1). La thiamine et la riboflavine sont dosées par voie microbiologique dans le foie, l'intestin et le reste de la carcasse à l'aide de milieux lyophilisés DrFco. Les méthodes employées sont les suivantes :

- pour la thiamine : technique de SARETt et CHeLdelin (1944) avec L. fermenti ATCC 9338,

- pour la riboflavine; technique de SNELI et STRONG (1939) avec L. casei ATCC 8or4.

Les modalités d'extraction sont les mêmes que celles qui ont été décrites par RtRAT (I960) et par BLuM (I966).

(1) Énergie $(k c a l)=(\mathrm{N} \times 6,25) \times 5,65 \times$ Lipides $\times 9,4^{n}$. 


\section{TABLEAU I B}

Composition des régimes

(Expériences $\mathrm{B}$ et $\mathrm{C}$ )

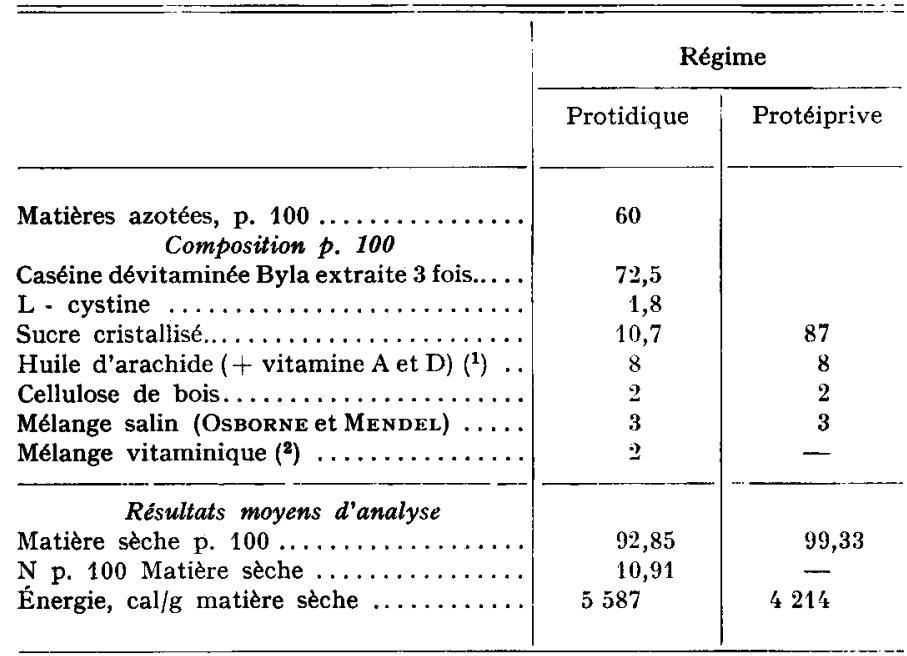
régime.

(1) A raison de 16 UI de vitamine $A$ et 3,2 UI de vitamine $D_{2}$ par $g$ de

(2) Mélange apportant les quantités suivantes de thiamine en $\mu \mathrm{g}$ par $\mathrm{g}$ de régime protidique : régime préexpérimental, néant ; lot A, 16 ; Lot $\mathrm{B}, 1,6$; Lot $\mathrm{C}, 0,08$. Les quantités des autres vitamines en $\mu \mathrm{g}$ par $\mathrm{g}$ de régime sont : pyridoxine, 16 ; riboflavine, 30 ; Pantothénate de $\mathrm{Ca}, 60$; Vitamine $\mathrm{B}_{12}, 0,06$; Acide folique, 20 ; Inisotol, 1000 ; Acide paraminobenzoïque, 1000 ; Vitamine E, 60 ; Vitamine K, 10 ; Biotine, 0,4 ; Choline, 2000.

TABLEAU 2

Précision du dosage des vitamines

\begin{tabular}{|c|c|c|c|c|}
\hline & \multicolumn{4}{|c|}{ Vitamine } \\
\hline & \multicolumn{2}{|c|}{ Thiamine } & \multicolumn{2}{|c|}{ Riboflavine } \\
\hline Tissu $\ldots \ldots \ldots \ldots \ldots \ldots \ldots \ldots \ldots \ldots$ & Foie & Carcasse & Foie & Carcasse \\
\hline Nombre d'échantillons $\ldots \ldots \ldots \ldots \ldots$ & 23 & 22 & 23 & 20 \\
\hline Moyenne, $\mu \mathrm{g} / g$ poids frais $\ldots \ldots \ldots \ldots$ & 6,70 & 1,34 & 10,39 & 3,10 \\
\hline Écart-type $\ldots \ldots \ldots \ldots \ldots \ldots \ldots \ldots$ & 0,93 & 0,15 & 1,23 & 0,51 \\
\hline 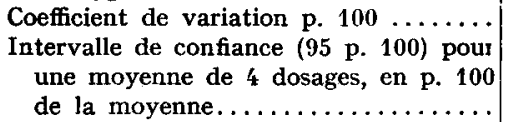 & $\pm 13,6$ & $\pm 10,9$ & $\pm 11,6$ & $\pm 16,1$ \\
\hline
\end{tabular}


Les dosages, pour la riboflavine, sont effectués en double exemplaire, à raison de deux échantillons par animal. Les résultats sont exprimés, pour la thiamine, en chlorhydrate de thiamine, et pour la riboflavine, sous forme de vitamine pure. La précision des dosages, selon la nature de la vitamine ou de l'échantillon, est indiquée dans le tableau $2:$ l'intervalle de confiance, pour une probabilité de $95 \mathrm{p}$. Ioo, varie entre ro et $15 \mathrm{p}$. 10o environ de part et d'autre de la valeur moyenne, obtenue à partir de deux échantillons dosés chacun en double exemplaire.

\section{Expérience B: Influence d'une carence simple en thiamine}

Soixante-dix rats, âgés de $2 \mathrm{r}$ à 23 jours et pesant en moyenne $50 \mathrm{~g}$, sont divisés en deux groupes au cours d'une période préexpérimentale. Un premier groupe de 60 animaux est soumis à une carence prolongés en thiamine jusqu'à l'obtention d'un arrêt de croissance et même d'une légère perte de poids. A cet effet, on leur administre, simultanément et dans deux mangeoires séparées :

- une quantité limitée de protéines $(\mathrm{I}, 8 \mathrm{~g} / \mathrm{j})$, à raison de $3 \mathrm{~g}$ d'un régime renfermant $60 \mathrm{p}$. 100 de protéines sous forme de caséine dévitaminée + cystine et additionné de toutes les vitamines, sauf la thiamine, à des doses couvrant largement le besoin de croissance ;

- un régime protéiprive à base de sucre alloué ad libitum.

Un lot témoin ( $\mathrm{T}$ ) de ro animaux est nourri dans les mêmes conditions que précédemment à l'aide d'un régime normalement supplémenté en thiamine.

A la différence de l'expérience précédente, les animaux sont soumis dès le début aux repas séparés afin de les habituer d'une façon progressive à ce mode d'alimentation et d'éviter ainsi une hétérogénéité trop grande dans l'ingestion des protéines en début d'expérience.

A l'issue de cette période, qui a duré 21 jours, 5 lots de 12 rats sont constitués suivant le dispositif des blocs complets "randomisés", d'après le poids, le gain de poids et la consommation pendant la période préexpérimentale.

Un lot témoin (TC) est sacrifié immédiatement, tandis que les quatre autres (I B, II B, III B, IV B) sont soumis à l'alimentation séparée selon les mêmes modalités que précédemment : $1,8 \mathrm{~g}$ de protéines/j sous forme de caséine dévitaminée, et régime protéiprive ad libitum.

Le régime protidique, qui renferme 60 p. roo de protéines est distribué à raison de $3 \mathrm{~g} / \mathrm{j}$. Il est additionné de doses variables de chlorhydrate de thiamine : une dose normale couvrant largement le besoin, soit : I6 $\mu$ g (lot I B) ; le I/ro de cette dose : I, 6( $\mu$ g lot II B) ; et le I/20 : 0,08 $\mu \mathrm{g}$ (lot III B). Les apports journaliers correspondants sont respectivement de $48,4,8$ et $2,4 \mu \mathrm{g}$. Toutes les autres vitamines sont fournies en excès par rapport aux besoins.

Dans le quatrième lot (IV B), les animaux reçoivent, à l'intérieur de chaque répétition, la même dose normale de thiamine que le lot $\mathrm{I} B$, soit $4^{8} \mu \mathrm{g} / \mathrm{j}$, et la même quantité d'énergie que celle ingérée la veille par le partenaire du lot II B, a fin de contrôler l'effet propre d'une carence en thiamine, indépendamment de son action sur l'appétit.

La composition des régimes, qui sont distribués à l'état sec, est rapportée dans le tableau I B. Les modalités expérimentales sont les mêmes que celles décrites dans l'expérience A. Notons cependant que la durée de l'expérience a dû être limitée à 14 jours en raison des difficultés rencontrées pour faire ingérer en totalité la dose journalière de régime protidique par les animaux les plus carencés en thiamine. A la fin de l'expérience, les animaux sont sacrifiés au gaz d'élairage. Le foie est séparé du reste de la carcasse et sur ces deux fractions on procède, comme dans l'expérience précédente, à l'analyse chimique globale : matière sèche, cendres, azote, les lipides étant estimés par différence, la thiamine et la riboflavine sont dosées par voie microbiologique.

\section{Expérience $C:$ Influence d'une carence simple en riboflavine}

L'étude de l'influence d'une carence plus ou moins sévère en riboflavine sur la consommation spontanée d'énergie a fait l'objet d'une troisième expérience $C$, dans les mêmes conditions que celles précisées dans l'expérience $B$. Cinquante rats âgés de $2 \mathrm{I}-23$ jours et d'un poids initial de $46,5 \mathrm{~g}$ sont soumis, pendant une période préexpérimentale de ro jours, à une déplétion en riboflavine. A cet effet, on leur administre, en deux repas séparés et sous forme sèche :

- un régime protidique apportant $\mathrm{I}, 8 \mathrm{~g}$ de protéines sous forme de caséine dévitaminée + cystine, additionnée d'un mélange vitaminique dépourvu de riboflavine ;

- un régime protéiprive ad libitum.

A l'issue de cette période, 4 lots de 12 animaux sont constitués :

- trois lots reçoivent dans leur repas protidique : $\mathrm{r}, 8 \mathrm{~g}$ de protéines $/ \mathrm{j}$, sous forme de caséine dévitaminée et l'une des doses suivantes de riboflavine : une dose normale, soit : $90 \mu \mathrm{g} / \mathrm{j}$ dans le 
lot I C ; une dose réduite au I/Io $(9 \mu \mathrm{g})$ dans le lot II C, et une dose réduite au $\mathbf{I} / 20(4,5 \mu \mathrm{g})$ dans le lot III $\mathrm{C}$; toutes les autres vitamines sont fournies en quantités couvrant largement les besoins. Le régime protéiprive est offert dans tous les cas ad libitum;

- Un lot témoin initial (TC) est sacrifié immédiatement en vue de 1'analyse chimique globale et du dosage des vitamines.

La durée de l'expérience est de 28 jours. La composition des régimes protidique et protéiprive est la même que dans l'expérience $\mathrm{B}$. A la fin de l'expérience, les animaux sont sacrifiés après un jeûne de 8 heures. Le foie est séparé immédiatement du reste de la carcasse et on procède à l'analyse chimique corporelle, ainsi qu'au dosage de la thiamine et de la riboflavine par les méthodes décrites précédemment.

Dans chacune des trois expériences, les rétentions azotée et énergétique sont calculées à partir des résultats de l'analyse chimique corporelle, par différence entre la quantité finale dosée dans la carcasse entière et la quantité initiale estimée à partir du lot témoin abattu en début d'expérience. L'efficacité de la rétention est définie par le coefficient d'utilisation pratique ou CUP (selon Terrorne et Valla, I933):

$$
\text { CUP N (ou énergie) }=\frac{\text { Quantité retenue }}{\text { Quantité ingérée }} \times \text { Ioo }
$$

Comme pour l'azote et l'énergie, les quantités de vitamines retenues dans l'organisme sont obtenues par différence entre le contenu final dosé dans la carcasse entière et le contenu initial estimé à partir du lot témoin sacrifié en début d'expérience. Le coefficient de rétention apparent des vitamines est apprécié par rapport à la quantité ingérée ; en opérant de cette manière, il n'a pas été tenu compte des quantités synthétisées par la microflore intestinale, ni des quantités éliminées ou dégradées.

\section{RÉSULTATS}

\section{Croissance et consommation (tabl. $3 \mathrm{~A}, 3 \mathrm{~B}$ et $3 \mathrm{C}$ )}

Comme le montre la figure I, l'administration d'un régime totalement dépourvu de vitamines $\mathrm{B}$ (exp. A) ou simplement carencé en thiamine (exp. B) ou en riboflavine (exp. C) provoque un ralentissement de la croissance dès la première semaine, par rapport à un régime témoin non carencé. Si l'on prolonge cette période dé déplétion jusqu'à trois semaines, on aboutit finalement à un arrêt de la croissance et même à une perte de poids des animaux, accompagnée d'une diminution de la consommation spontanée d'énergie.

\section{TABLEAU $3 \mathrm{~A}$}

Résultats généraux de croissance et de consommation

(Expérience A)

Nombre de rats par lot : 9 Poids moyen initial: 49, I g Durée : 28 jours

\begin{tabular}{|c|c|c|c|c|}
\hline \multirow{3}{*}{ Apport de Vitamines $B \ldots \ldots \ldots \ldots \ldots$} & \multicolumn{4}{|c|}{ Lot } \\
\hline & I A & & II A & III A \\
\hline & Normal & & $1 / 10$ & $\mathrm{I} / 20$ \\
\hline Gain moyen $(\mathrm{g}) / \mathrm{j} \ldots \ldots \ldots \ldots \ldots \ldots \ldots$ & 2,68 土 $0,18 *$ & 1,52 & $\pm 0,10$ & $0,19 \pm 0,09$ \\
\hline $\mathrm{N}$ ingéré, $\mathrm{mg} / \mathrm{j} \ldots \ldots \ldots \ldots \ldots \ldots \ldots$ & $198 \pm 3,9$ & 193 & $\pm 1,4$ & $192 \pm 3,8$ \\
\hline Matière sèche ingérée, $\mathrm{g} / \mathrm{j} \ldots \ldots \ldots \ldots \ldots$ & $9,44 \pm 0,42$ & 6,58 & 土 0,32 & $4,96 \pm 0,20$ \\
\hline Énergie ingérée, $\mathrm{kcal} / \mathrm{j} \ldots \ldots \ldots \ldots \ldots \ldots$ & $41,38 \pm 1,67$ & 29,7 & $\pm 1,30$ & $23,18 \pm 0,81$ \\
\hline
\end{tabular}

* Écart-type de la moyenne. 
Si l'on considère les résultats des expériences proprement dites (tabl. $3 \mathrm{~A}, 3 \mathrm{~B}$ et $3 \mathrm{C}$ ), on constate que pour une ingestion azotée sensiblement identique dans les différents lots, la vitesse de croissance pendant 28 jours (exp. A et C) ou I 4 jours (exp. B) est d'autant plus faible que l'apport vitaminique est plus réduit. L'addition d'une dose normale de vitamines permet au rat de reprendre une vitesse de croissance au moins égale à celle du lot témoin n'ayant subi aucune carence $: 5,24 \mathrm{~g} / \mathrm{j}$ contre $4,36 \mathrm{~g}$ dans le cas de la thiamine.

TABLEAU $3 \mathrm{~B}$

Résultats de croissance et de consommation

Durée : I4 jours $\begin{aligned} & \text { (Expérience B) } \\ & \text { Poids moyen initial : 69, I g }\end{aligned}$

\begin{tabular}{|c|c|c|c|c|}
\hline \multirow{3}{*}{$\begin{array}{l}\text { Apport de thiamine } \ldots \ldots \ldots \ldots \ldots \\
\text { Energie... } \ldots \ldots \ldots \ldots \ldots \ldots \ldots \ldots\end{array}$} & \multicolumn{4}{|c|}{ Lot } \\
\hline & I B & II B & III B & IV $\mathrm{B}$ \\
\hline & $\begin{array}{l}\text { Normal } \\
A d \text { libitum }\end{array}$ & $\begin{array}{c}1 / 10 \\
\text { Ad libitum }\end{array}$ & $\begin{array}{c}1 / 20 \\
\text { Ad libitum }\end{array}$ & $\begin{array}{c}\text { Normal } \\
\text { Comme II B }\end{array}$ \\
\hline Nombre d'animaux $\ldots \ldots \ldots \ldots$ & 12 & 12 & 7 & 12 \\
\hline Gain moyen, $g / j \ldots \ldots \ldots \ldots$ & $5,24 \pm 0,18$ & $1,75 \pm 0,18$ & $0,45 \pm 0,11$ & $2,50 \pm 0,09$ \\
\hline $\mathbf{N}$ ingéré, $\mathrm{mg} / \mathrm{j} \ldots \ldots \ldots \ldots \ldots$ & $296 \pm 2,0$ & $288 \pm 3,7$ & $264 \quad \pm 11,7$ & $290 \pm 3,0$ \\
\hline Matière sèche ingérée, $g / j . \ldots \ldots$ & $12,15 \pm 0,51$ & $7,45 \pm 0,34$ & $5,51 \pm 0,31$ & $7,30 \pm 0,33$ \\
\hline Énergie ingérée, $\mathrm{kcal} / \mathrm{j} \ldots \ldots \ldots \ldots$ & $54,95 \pm 2,15$ & $35,06 \pm 1,45$ & $26,48 \pm 1,39$ & $34,44 \pm 1,44$ \\
\hline
\end{tabular}

\section{TABleaU $3 \mathrm{C}$}

Résultats généraux de croissance et de consommation

(Expérience $\mathrm{C}$ )

Poids moyen initial : 65,4 g Durée : 28 jours

Apport de riboflavine $\ldots \ldots \ldots \ldots \ldots \ldots \ldots$

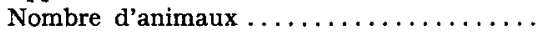

Gain moyen, $g / j \ldots \ldots \ldots \ldots \ldots \ldots \ldots$

$\mathrm{N}$ Ingéré, $\mathrm{mg} / \mathrm{j} \ldots \ldots \ldots \ldots \ldots \ldots \ldots \ldots$

Matière sèche ingérée, $g / j \ldots \ldots \ldots \ldots \ldots$

Énergie ingérée, $\mathrm{kcal} / \mathrm{j}$..

\begin{tabular}{|c|c|c|}
\hline \multicolumn{3}{|c|}{ Lot } \\
\hline I C & II C & III C \\
\hline Normal & $1 / 10$ & $1 / 20$ \\
\hline 12 & 11 & 6 \\
\hline $4,43 \pm 0,10$ & $3,06 \pm 0,08$ & $2,41 \pm 0,12$ \\
\hline $299 \pm 0,2$ & $290 \pm 4,2$ & $293 \pm 2,3$ \\
\hline $14,12 \pm 0,35$ & $10,59 \pm 0,30$ & $9,77 \pm 0,55$ \\
\hline $63,62 \pm 1,49$ & $48,31 \pm 1,26$ & $44,95 \pm 2,30$ \\
\hline
\end{tabular}

La croissance reprend également, quoique plus lentement, avec une dose représentant le I/Io de la précédente, tandis qu'avec une dose réduite au I/20, la dépression de croissance est encore plus accentuée. Ceci est particulièrement net dans le cas d'une carence globale en vitamines B et d'une carence simple en thiamine ; le poids des ani- 
maux demeure pratiquement inchangé pendant toute la durée de l'expérience. Il est à noter qu'à mesure que s'accentue la carence vitaminique, il est de plus en plus difficile de faire ingérer par les rats la totalité du régime protidique ; ceci explique

croissance

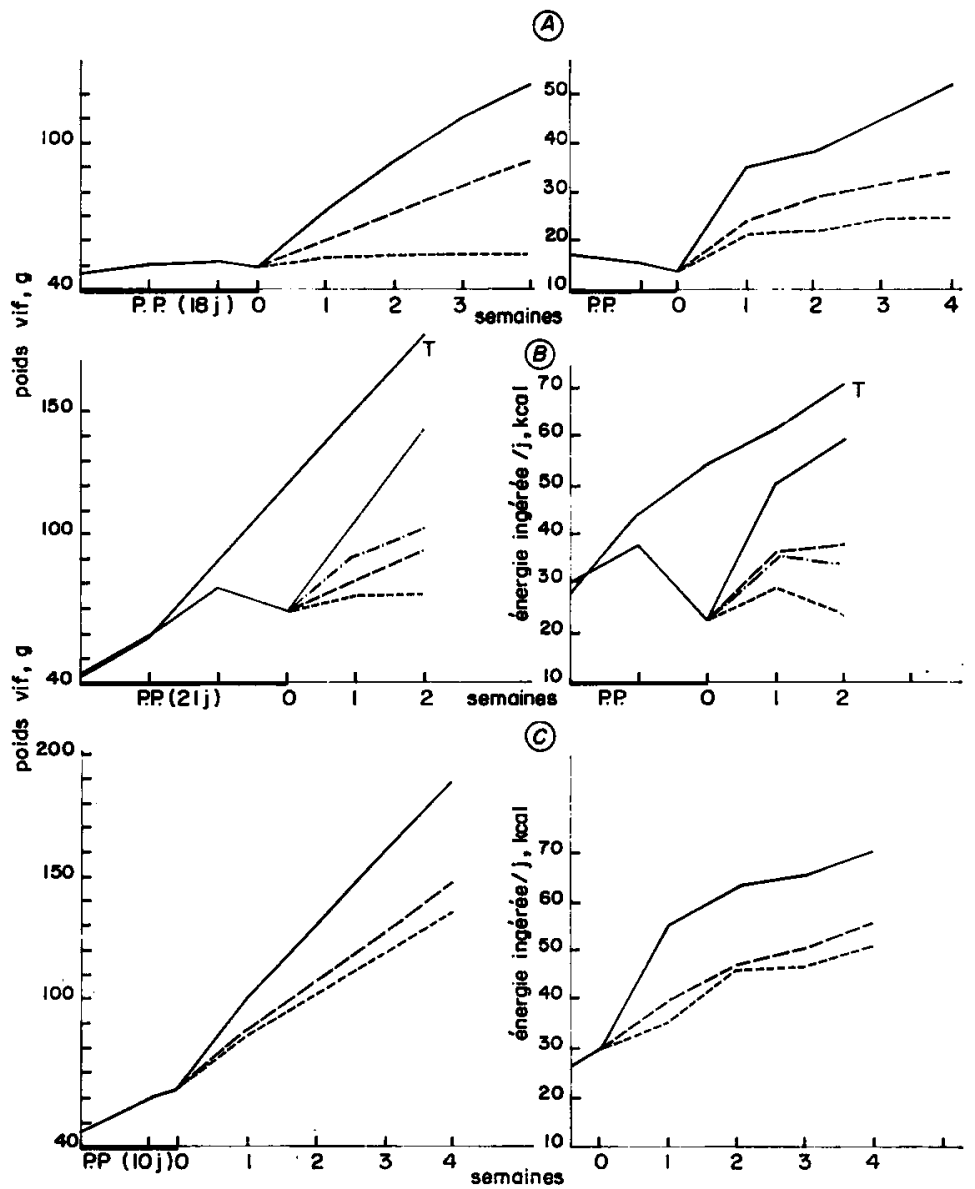

Fig. I. - Évolution de la croissance et de la consommation d'énergie

a) Croissance

b) Consommation d'énergie

Exp. A. Polycarence en vitamines B

Exp. B. Carence en thiamine

Exp. C. Carence en riboflavine

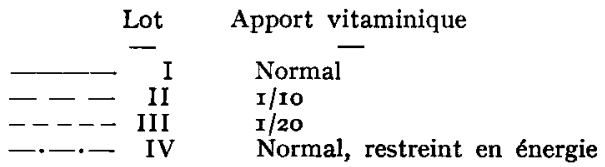

P. P. : période préexpérimentale. $\mathrm{T}$ : Lot témoin non carencé initialement.

qu'un certain nombre d'animaux ont dû être éliminés. Par ailleurs, la comparaison des lots III et IV de l'expérience $B$ montre qu'à niveau d'ingestion azotée et énergétique égal, un déficit en thiamine provoque une dépression sensible de la croissance. 
La consommation spontanée d'énergie suit une évolution sensiblement parallèle à celle de la vitesse de croissance. Ainsi, les animaux des lots les plus carencés en vitamines B (III A) ou en thiamine (III B) n'ont pratiquement pas varié de poids ou de consommation, tandis que dans les autres lots, la quantité d'énergie ingérée est d'autant plus importante que le gain de poids est lui-même plus élevé (fig. I). L'évolution de la consommation d'énergie en fonction du poids moyen par lot et par semaine (fig. 2), fait de plus apparaître une relation de type exponentiel, mais elle demeure
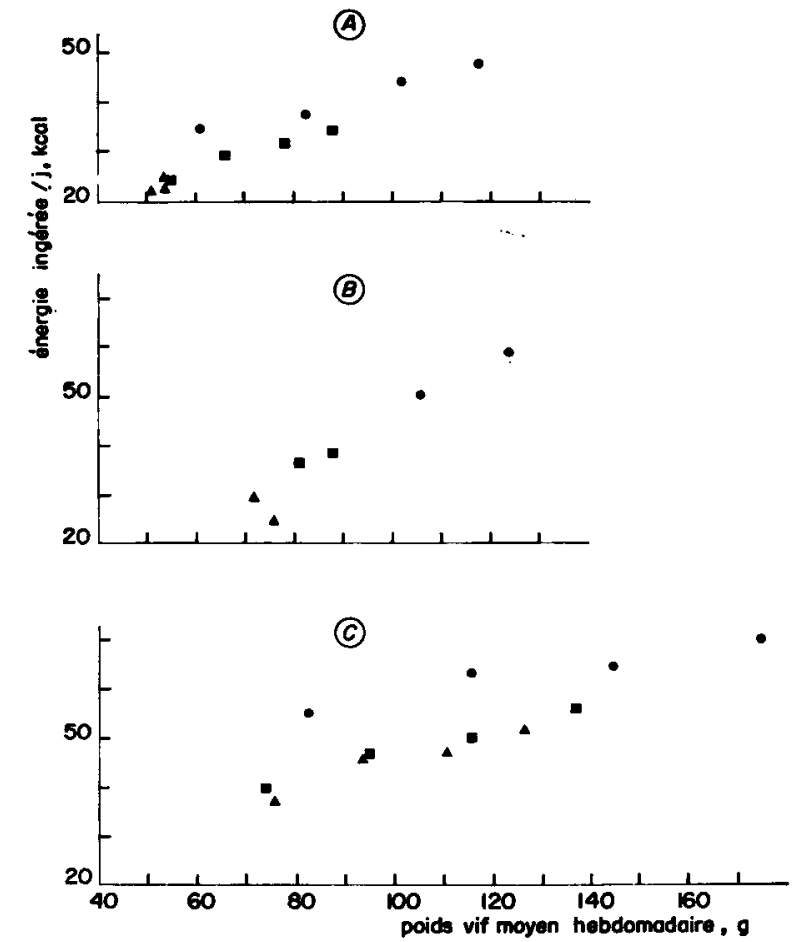

FIG. 2. - Evolution de la consommation spontanée d'énergie

en fonction du poids vif moyen hebdomadaire (résultats moyens par lot)

Jixp. A. Polycarence en vitamines B Exp. B. Carence en thiamine Exp. C. Carence en riboflavine Lot Apport vitaminique

$\begin{array}{rrl}\bullet & \text { I } & \text { Normal } \\ \bullet & \text { II } & \text { I-IO } \\ \mathbf{4} & \text { III } & \text { I } / 20\end{array}$

plus élevée, à un poids donné, dans les lots qui ont reçu une dose normale de vitamines ; la réalité de cette relation exponentielle a été démontrée dans une publication antérieure (HENRY et RÉRAT, I964).

\section{Composition corporelle}

a) Composition chimique globale (tabl. $4 \mathrm{~A}, 4 \mathrm{~B}$ et $4 \mathrm{C}$ ).

Les effets d'une polycarence en vitamines B (exp. A) ou de carences simples en thiamine (exp. B) et en riboflavine (exp. C), se caractérisent dans l'ensemble par une 
diminution importante des pourcentages de matière sèche et de lipides dans la carcasse entière, tandis que les teneurs en protéines et en cendres augmentent. Remarquons cependant qu' au niveau des organes (foie, intestin), les modifications sont sensiblement différentes. Ainsi, la diminution du poids du foie, sous l'influence d'un apport insuffisant de vitamines, est accompagnée d'une élévation de la concentration de la

\section{TABLEAU $4 \mathrm{~A}$}

Résultats de composition corporelle

(Expérience A)

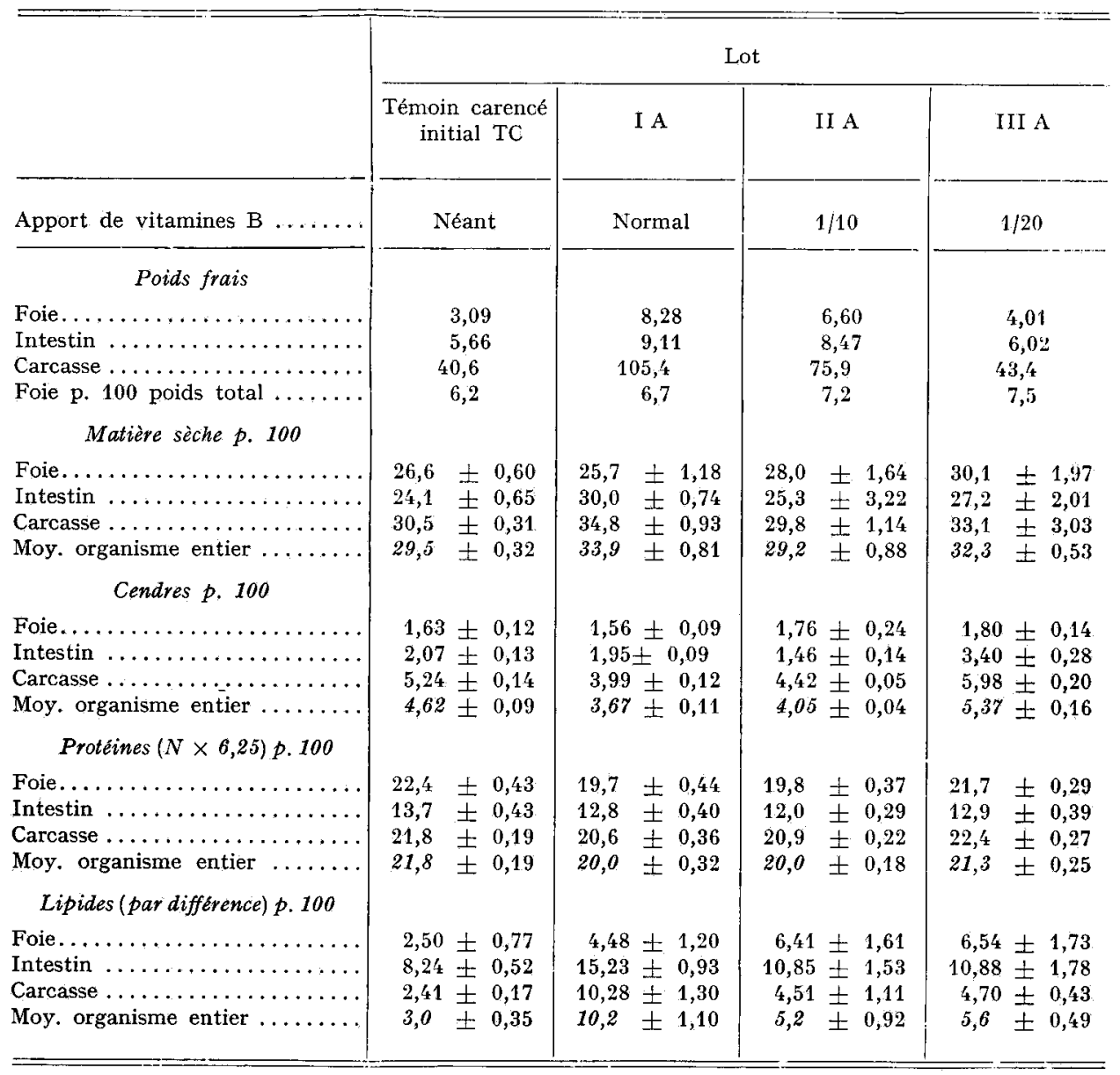

matière sèche, des cendres, des protéines et des lipides par rapport au poids frais. A l'inverse, dans l'intestin (exp. A), les pourcentages de matière sèche et de lipides sont en constante diminution, alors que la teneur en protéines est pratiquement inchangée et que le pourcentage de cendres augmente. Il convient de signaler que dans l'estimation des lipides par différence, il n'a pas été tenu compte du glycogène, dont l'impor- 


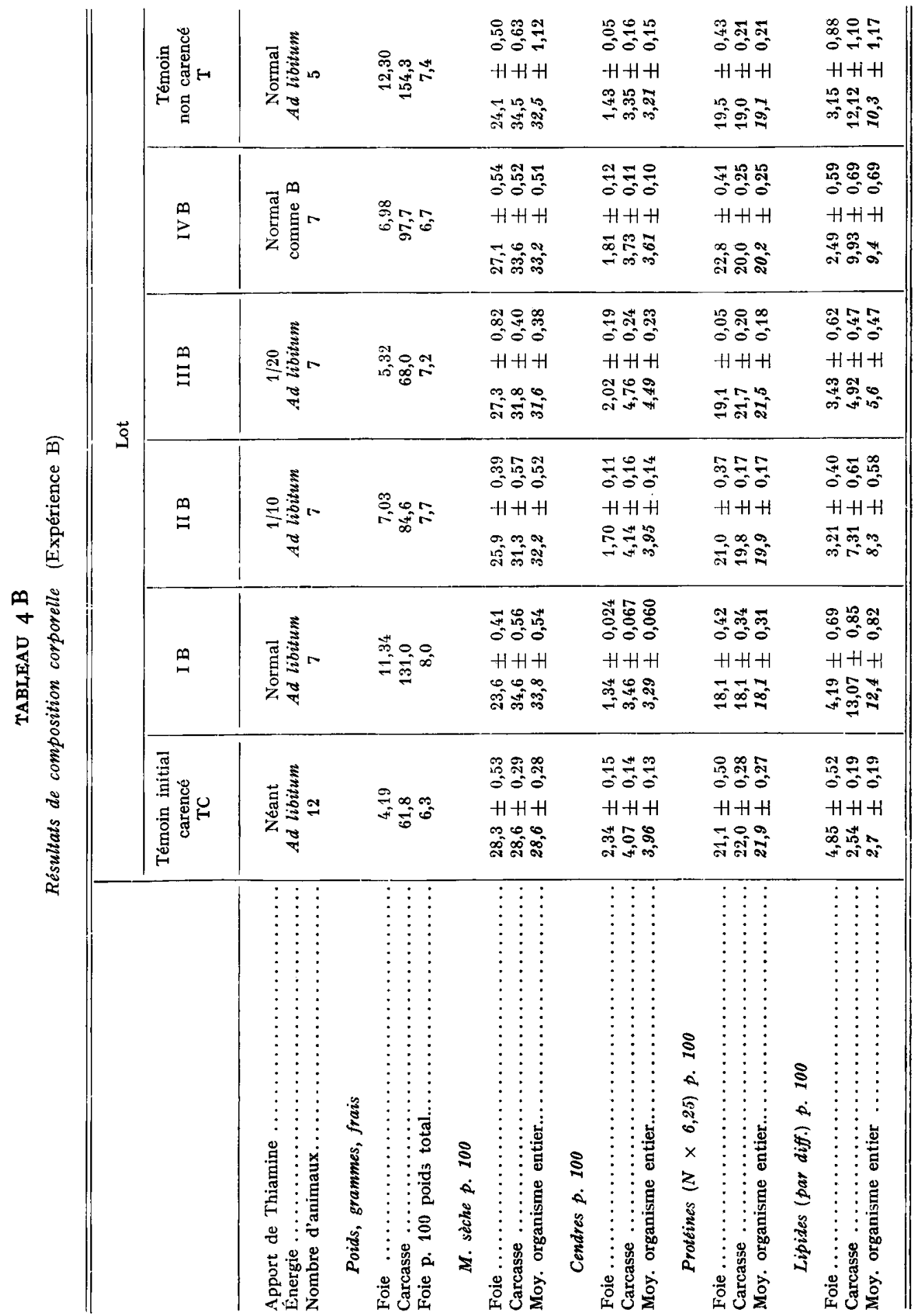


tance dans le foie n'est pas négligeable. Notons par ailleurs que dans chacune des trois expériences, le lot carencé, sacrifié au début de l'expérience, présente sensiblement les mêmes caractéristiques corporelles que le lot III qui a reçu le I/zo de la dose normale et s'est maintenu sensiblement au même poids pendant toute la durée de l'expérience.

\section{TABLEAU $4 \mathrm{C}$}

Résultats de composition corporelle

(Expérience $\mathrm{C}$ )

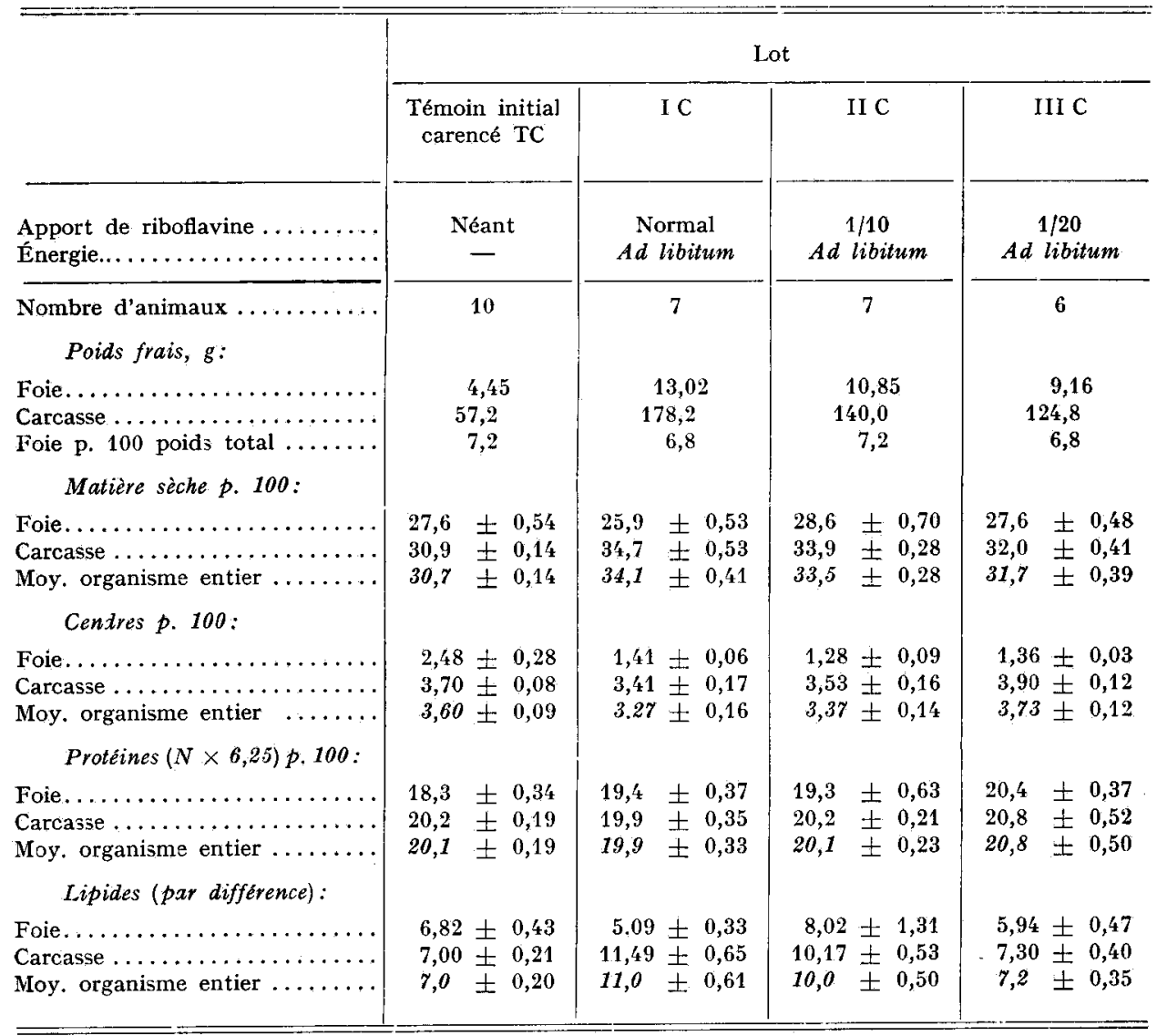

b) Bilans azoté et énergétique (tabl. $5 \mathrm{~A}, 5 \mathrm{~B}$ et $5 \mathrm{C}$ ).

La réduction de l'apport vitaminique provoque, d'une manière générale, une diminution importante des quantités d'azote et d'énergie retenues, que ce soit en valeur absolue (rétention journalière) ou en valeur relative (coefficient d'utilisation pratique). Si l'on examine de plus près les résultats de l'expérience $B$, on constate qu'à consommation d'énergie égale,une réduction de la dose journalière de thiamine de $48 \mu$ (lot IV) à $4,8 \mu \mathrm{g}$ (lot II), provoque à la fois une diminution du dépôt azoté et de la rétention 
énergétique. Puisque la part de l'entretien dans la consommation totale a été sensiblement la même dans les deux lots, on en déduit qu'une carence en thiamine excrce une action dépressive sur l'utilisation nette des éléments énergétiques pour la croissance,

\section{TABLEAU $5 \mathrm{~A}$}

Bilans azoté et énergétique (Expérience A)

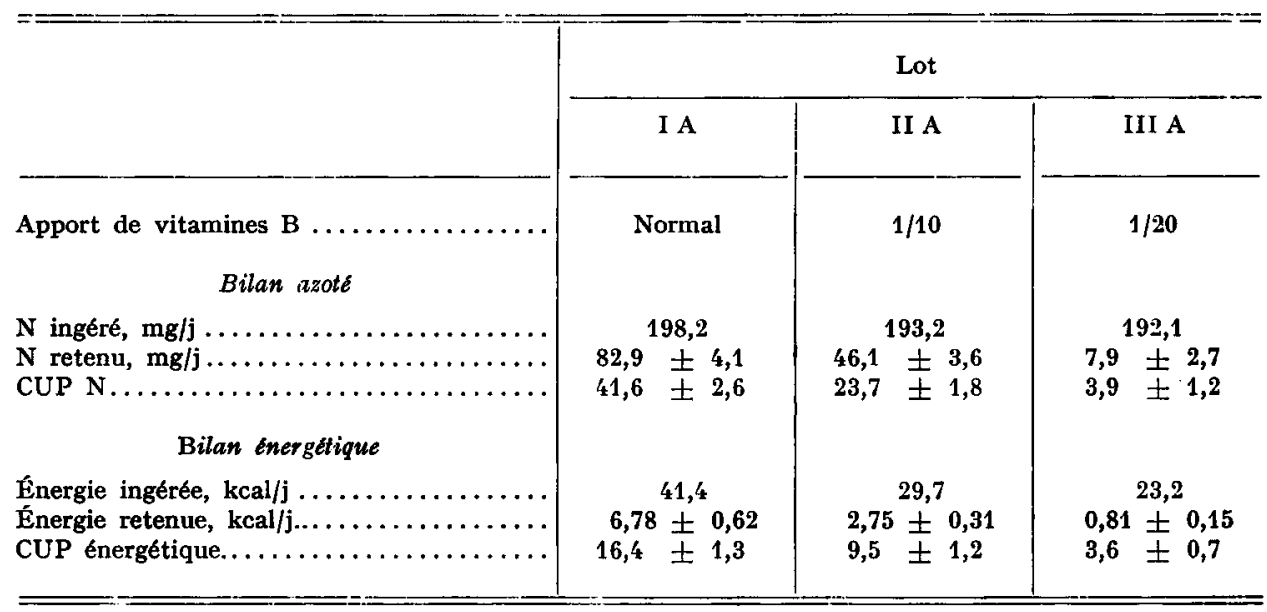

TABLEAU 5 B

Bilans azoté et énergétique (Expérience B)

\begin{tabular}{|c|c|c|c|c|}
\hline & \multicolumn{4}{|c|}{ Lot } \\
\hline & I B & II B & III B & IV B \\
\hline $\begin{array}{l}\text { Apport de thiamine } \ldots \ldots \ldots \ldots \ldots \\
\text { Énergie }\end{array} \ldots \ldots \ldots \ldots \ldots \ldots \ldots \ldots$ & $\begin{array}{l}\text { Normal } \\
\text { Ad libitum }\end{array}$ & $\begin{array}{l}\mathrm{I} / \mathbf{1 0} \\
\text { Ad libitum }\end{array}$ & $\begin{array}{c}1 / 20 \\
\text { Ad libitum }\end{array}$ & $\begin{array}{l}\text { Normal } \\
\text { Ad libitum }\end{array}$ \\
\hline $\begin{array}{l}\qquad \begin{array}{c}\text { Bilan azoté } \\
\mathrm{N} \\
\mathrm{N} \text { ingéré, } \mathrm{mg} / \mathrm{j} \ldots \ldots \ldots \ldots \ldots \ldots\end{array} \\
\mathbf{N} \text { retenu, } \mathrm{mg} / \mathrm{j} \ldots \ldots \ldots \ldots \ldots \ldots \ldots\end{array}$ & $\begin{array}{r}294,4 \\
120,9 \quad 1 \quad 11,0 \\
41,0 \text { 土 } 3,6\end{array}$ & \begin{tabular}{c}
\multicolumn{2}{c}{284,1} \\
$33,7 \pm 7,5$ \\
$11,9 \pm 2,6$
\end{tabular} & \begin{tabular}{rr}
\multicolumn{2}{c}{268,7} \\
20,1 & $\pm 4,9$ \\
7,3 & $\pm 1,6$
\end{tabular} & \begin{tabular}{l}
\multicolumn{2}{c}{290,4} \\
$67,1 \quad$ 土 7,5 \\
23,0 土 2,4
\end{tabular} \\
\hline $\begin{array}{l}\qquad \text { Bilan énergettique } \\
\text { Énergie ingérée, } \mathrm{kcal} / \mathrm{j} \ldots \ldots \ldots \\
\text { Énergie retenue, } \mathrm{kcal} / \mathrm{j} \ldots \ldots \ldots \\
\text { C.U.P. Énergétique } \ldots \ldots \ldots \ldots \ldots\end{array}$ & $\begin{array}{c}56,86 \\
14,77 \pm 0,90 \\
26,0 \pm 1,3\end{array}$ & $\begin{array}{c}35,07 \\
5,06 \pm 0,86 \\
14,0 \pm 1,7\end{array}$ & $\begin{array}{l}23,41 \\
1,63 \pm 0,30 \\
5,0 \pm 1,1\end{array}$ & $\begin{array}{c}35,16 \\
7,66 \pm 0,87 \\
21,5 \pm 1,3\end{array}$ \\
\hline
\end{tabular}

en dehors d'une action dépressive sur 1'appétit pour les substances énergétiques. Par ailleurs, de la comparaison des lots $I$ et IV de l'expérience $B$, il ressort qu'une restriction énergétique de l'ordre de 37 p. Ioo provoque non seulement un dépôt moindre 
d'énergie, mais également une moins bonne utilisation de la fraction azotée ; par contre, le coefficient d'utilisation de l'énergie est sensiblement le même dans les deux lots.

TABLEAU $5 \mathrm{C}$

Bilans azoté et énergétique

(Expérience $\mathrm{C}$ )

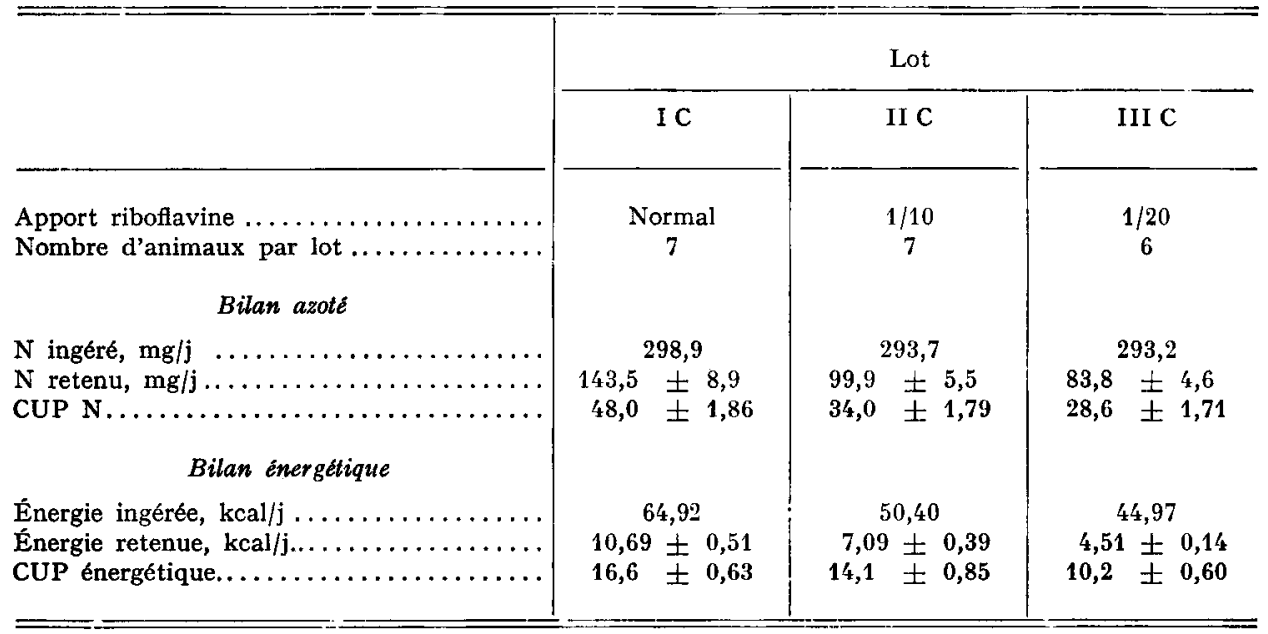

L'évolution de la consommation moyenne d'énergie par lot, en fonction de la quantité d'azote fixée dans les tissus, fait apparaître, pour l'ensemble des trois expériences (fig. 3), une relation linéaire suivant l'équation de régression : $\mathrm{E}_{\mathrm{I}}(\mathrm{kcal} / \mathrm{j})=\mathrm{I} 8,74+0,309 \mathrm{~N}(\mathrm{mg} / \mathrm{j})$, le coefficient de corrélation étant $r=0,976$.

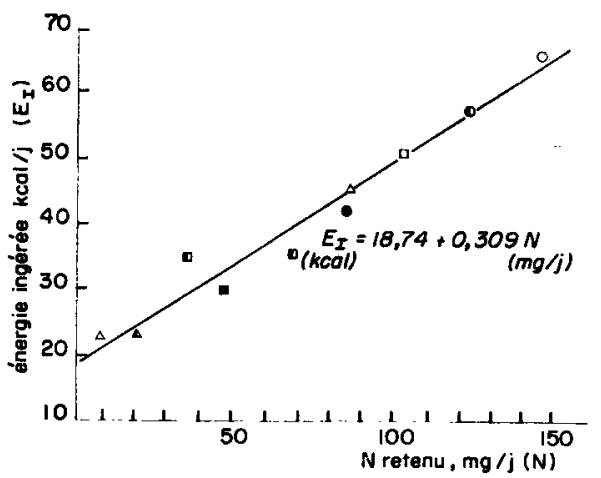

FIG. 3. - Relation entre la consommation spontanée d'énergie et la rétention azotée Apport vitaminique

\begin{tabular}{cccc} 
Exp. & Normal & I/Io & I/20 \\
\hline A & - & - & - \\
B & $\vdots$ & $\square$ & $\Delta$ \\
C & $\square$ & 0 & $\Delta$
\end{tabular}


$\mathrm{L}_{\mathrm{a}}$ valeur du coefficient de régression ( $309 \mathrm{kcal}$ par $\mathrm{g}$ d'N retenu) est sensiblement la même que celle trouvée antérieurement en faisant varier simplement l'apport azoté (RÉRAT, HENRY et JACQUOT, I963).

c) Répartition des vitamines dans l'organisme.

Polycarence en vitamines $B$ (tabl. $6 \mathrm{~A}$ ).

Afin de suivre la distribution des vitamines dans les organes et tissus, nous avons choisi parmi celles-ci les deux qui nous semblaient les plus représentatives, la thiamine

TABLEAU $6 \mathrm{~A}$

Répartition des vitamines dans l'organisme

(Expérience A)

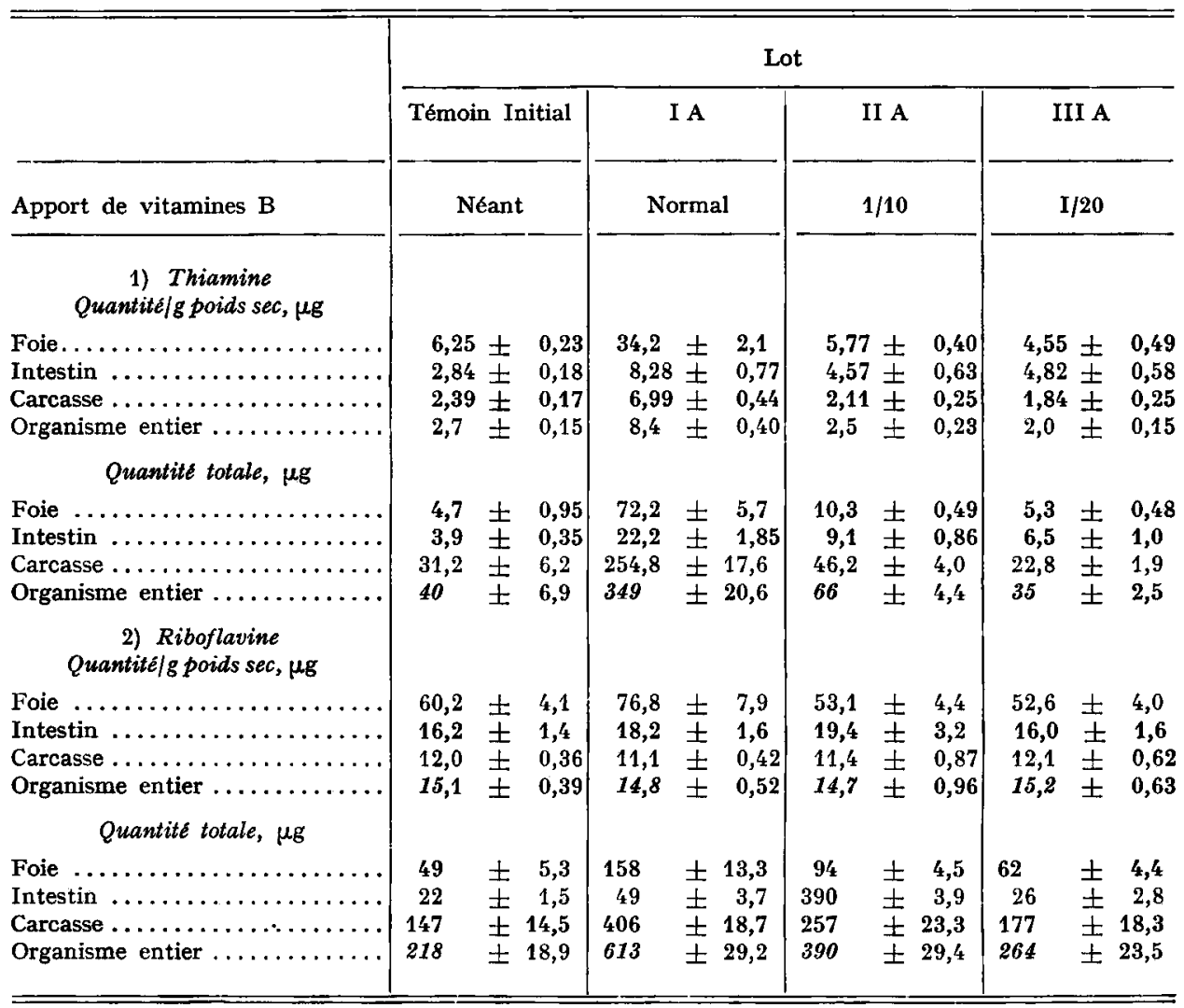

et la riboflavine. Des résultats du tableau $5 \mathrm{~A}$, il ressort que sous l'effet d'une carence, la quantité de thiamine par $\mathbf{g}$ de poids sec diminue considérablement pour atteindre une limite asymptotique au-delà du dixième de la dose normale $(5,3 \mu \mathrm{g} / \mathrm{j})$, alors que la quantité de riboflavine demeure sensiblement constante. 


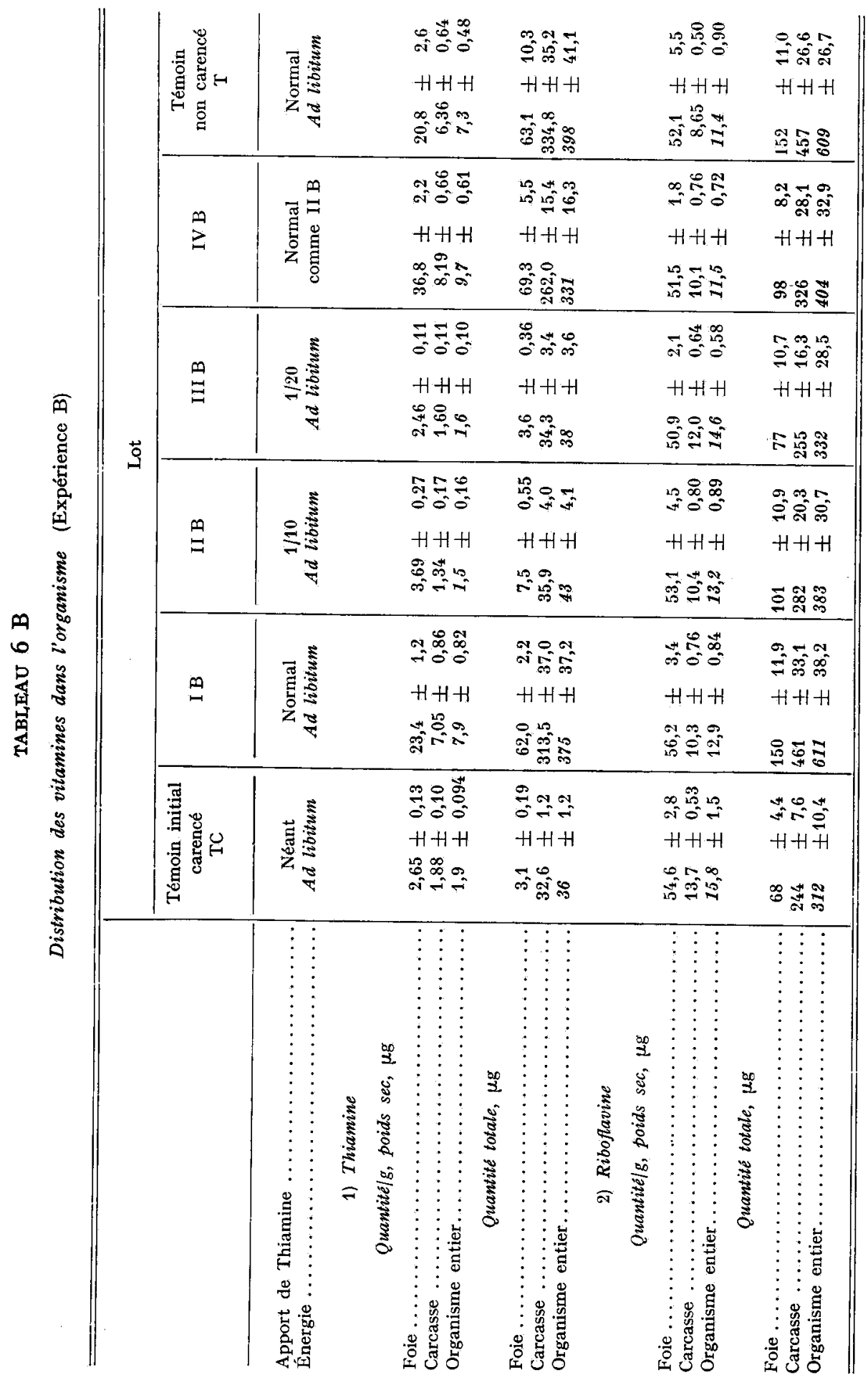


La diminution de la teneur en thiamine apparaît encore avec plus de netteté dans le foie (de $34,2 \mu \mathrm{g}$ à 5,8 et $4,5 \mu \mathrm{g} / \mathrm{g}$ respectivement pour $53-5,3$ et $2,7 \mu \mathrm{g}$ de thiamine $/ \mathrm{j}$ ) ; la teneur en riboflavine subit également une légère diminution de $77 \mu \mathrm{g}$ à $53 \mu \mathrm{g} / \mathrm{g}$ de poids sec environ.

Dans l'intestin et son contenu, on assiste de la même façon à une chute des concentrations de vitamines par rapport au poids sec. Cette chute est surtout importante dans le cas de la thiamine dont la concentration passe de 8,3 à $4,8 \mu \mathrm{g} / \mathrm{g}$; par contre; dans le cas de la riboflavine, elle est beaucoup moins brutale (de 18,2 à $16 \mu \mathrm{g} / \mathrm{g}$ ). Ceci semble indiquer qu'une carence sévère en thiamine ou en riboflavine n'est pas compensée par une augmentation de la synthèse par la microflore intestinale.

Carence en thiamine (tabl. $6 \mathrm{~B}$ ).

La comparaison des teneurs en vitamines dans le foie et la carcasse conduit aux mêmes constatations que pour une polycarence en vitamines du groupe $B$. Ainsi, à mesure que la sévérité de la carence en thiamine s'accentue, la concentration de cette dernière dans le foie diminue très fortement de 23,4 à $2,5 \mu \mathrm{g} / \mathrm{g}$ de poids sec, cependant

TABLEAU $6 \mathrm{C}$

Distribution des vitamines dans l'organisme

(Expérience $\mathrm{C}$ )

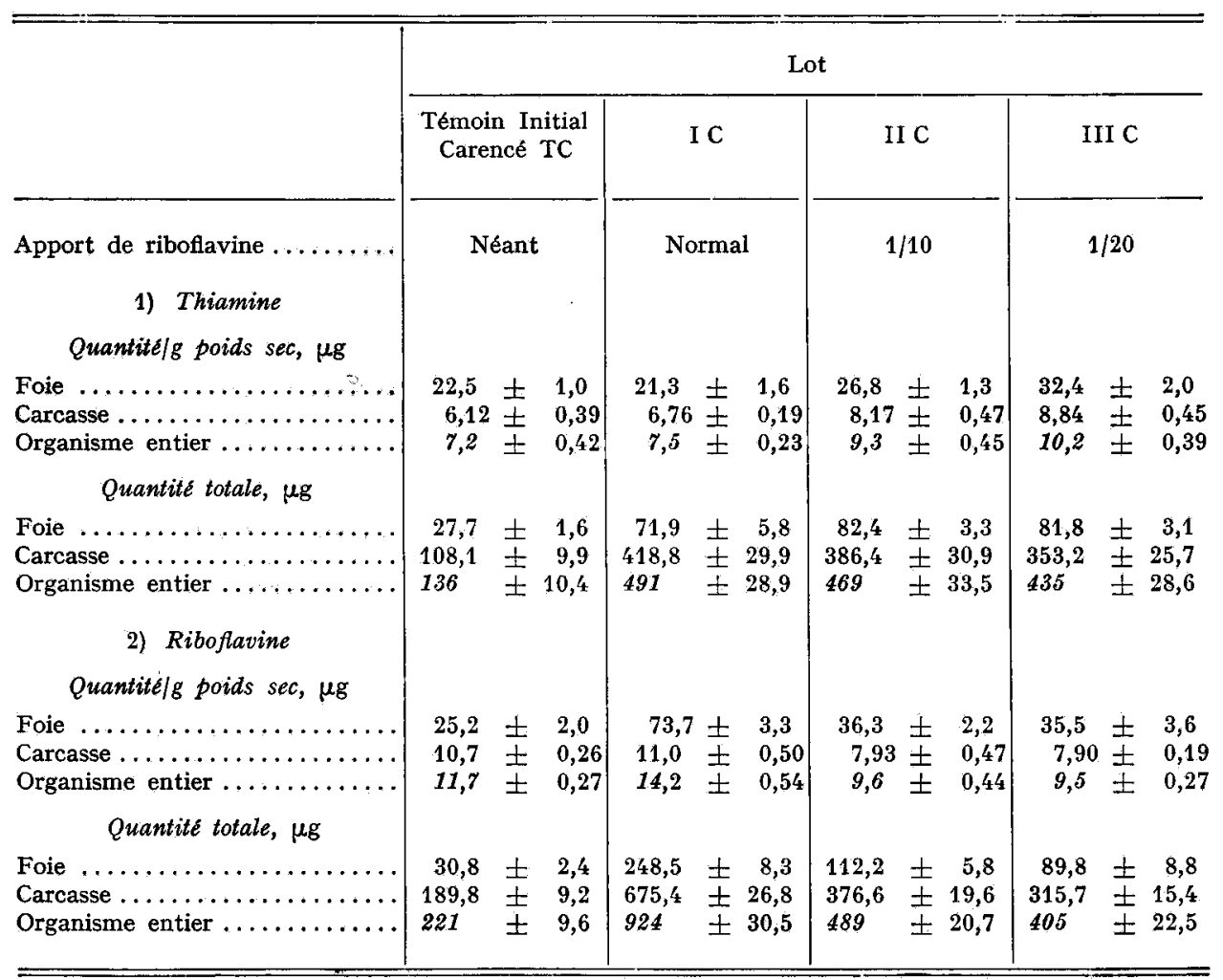


que dans la carcasse, elle atteint une valeur minimum dès la dose représentant le I/ro de la dose normale (lot II B) ; ce taux minimum est le même que celui enregistré dans le lot témoin carencé initial (TC).

Par ailleurs, si l'on considère le lot IV $B$ qui a reçu la même dose journalière (" normale ") de thiamine que le lot I B, mais dont le poids final est plus faible (I03 g contre $I_{42} \mathrm{~g}$ ) en raison d'une restriction énergétique de $37 \mathrm{p}$. Ioo, on remarque que les teneurs en thiamine sont notablement accrues dans le foie et la carcasse. En réalité, les quantités totales de thiamine dans le foie sont sensiblement les mêmes dans les deux lots (respectivement 69 et $62 \mu \mathrm{g}$ ), et les quantités présentes dans l'organisme entier sont peu différentes entre elles (respectivement 33I et $375 \mu \mathrm{g}$ ). Contrairement à ce que l'on observe pour la thiamine, l'évolution des teneurs en riboflavine ne fait apparaître aucune différence sensible d'un lot à l'autre, qu'il s'agisse du foie ou de la carcasse.

Carence en riboflavine (tabl. $6 \mathrm{C}$ ).

L'administration d'un régime carencé en riboflavine provoque une diminution importante de la teneur en cette vitamine dans le foie et la carcasse. Lorsqu'on réduit l'apport au $\mathbf{I} / \mathbf{I o}$ de la dose normale, les quantités de riboflavine par $\mathrm{g}$ de tissu sec passent de 74 à $36 \mu \mathrm{g}$ pour le foie et de II à $8 \mu \mathrm{g}$ pour le reste de la carcasse. Au-delà de ce seuil, la concentration des tissus en riboflavine se maintient sensiblement au même niveau.

En ce qui concerne la thiamine, au contraire, on constate une augmentation sensible des quantités par $\mathrm{g}$ de poids frais dans le foie et la carcasse, à mesure que la carence en riboflavine devient plus sévère. Cette élévation de la concentration de la

TABLEAU $7 \mathrm{~A}$

Bilans vitaminiques

(Expérience A)

\begin{tabular}{|c|c|c|c|}
\hline & \multicolumn{3}{|c|}{ Lot } \\
\hline & I A & II A & III A \\
\hline $\begin{array}{l}\text { Apport de vitamines } \mathrm{B} \ldots \ldots \ldots \ldots \ldots \ldots \\
\text { Thiamine }\end{array}$ & Normal & $1 / 10$ & $1 / 20$ \\
\hline $\begin{array}{l}\text { Quantité ingérée, } \mu g / \mathrm{j} \ldots \ldots \ldots \ldots \ldots \ldots \ldots \ldots \ldots \ldots \ldots \ldots \ldots \ldots \\
\text { Quantité retenue, } \mu g / \mathrm{j} \ldots \ldots \ldots \ldots \ldots \ldots \ldots \\
\text { Rétention apparente, p. } 100 \ldots \ldots \ldots \ldots\end{array}$ & $\begin{array}{l}53,3 \\
11,25 \pm 0,74 \\
21,6 \pm 1,4\end{array}$ & $\begin{aligned} 5,3 & \\
1,04 & \pm 0,18 \\
20,6 & \pm 3,5\end{aligned}$ & $-\stackrel{2,7}{0,10} \pm 0,12$ \\
\hline Riboflavine & & & \\
\hline $\begin{array}{l}\text { Quantité ingérée, } \mu g / \mathrm{j} \ldots \ldots \ldots \ldots \ldots \ldots \ldots \\
\text { Quantité retenue, } \mu \mathrm{g} / \mathrm{j} \ldots \ldots \ldots \ldots \ldots \ldots \ldots \ldots \\
\text { Rétention apparente p. } 100 \ldots \ldots \ldots \ldots \ldots \ldots\end{array}$ & $\begin{array}{l}100,0 \\
14,71 \pm 0,72 \\
15,1 \pm 1,1\end{array}$ & $\begin{array}{l}10,0 \\
6,61 \pm 0,71 \\
69,9 \pm 7,8\end{array}$ & $\begin{array}{l}5,0 \\
2,18 \stackrel{ \pm, 57}{ \pm} \\
46,7 \pm 11,7\end{array}$ \\
\hline
\end{tabular}

thiamine dans les tissus est due en réalité à une diminution de poids des animaux, les quantités totales dans le foie et le reste de la carcasse étant sensiblement les mêmes d'un lot à l'autre. 


\section{d) Bilans vitaminiques.}

Io Sous l'effet d'une carence globale en vitamines (tabl. 7 A), le bilan de thiamine diminue très fortement dans le lot II (I/Io de la dose normale) et devient même négatif dans le lot III ( $\mathrm{I} / 20$ de la dose normale). Le pourcentage de thiamine retenue dans l'organisme est pratiquement inchangé (environ $20 \mathrm{p}$. Ioo), lorsque l'apport de vitamines varie dans le rapport de I à Io. D'après les résultats précédents, il apparaît ainsi que le dépôt de thiamine dans les tissus, et principalement dans le foie, croît à un rythme sensiblement proportionnel à l'apport du régime, à partir d'un apportseuil de $5 \mu \mathrm{g} / \mathrm{j}$, qu'on pourrait considérer comme le besoin minimum du rat en thiamine ; ceci confirme l'estimation faite antérieurement par MORGAN et YUDKIN (I962).

Comme pour la thiamine, la quantité de riboflavine fixée par les tissus diminue très fortement lorsqu'on réduit l'apport de cette vitamine, en même temps que les autres vitamines du groupe $B$, mais demeure positive au niveau minimum. En valeur relative, la rétention est maximum avec un apport de $10 \mu \mathrm{g} / \mathrm{j}$ (70 p. Ioo), mais présente encore une valeur élevée au niveau inférieur đe $5 \mu \mathrm{g} / \mathrm{j}$ (20 p. roo), tandis qu'à la dose normale de $100 \mu \mathrm{g} / \mathrm{j}$, une quantité importante de la vitamine est excrétée ou détruite.

$2^{\circ}$ Une carence simple en thiamine (tab1. 7 B) provoque les mêmes effets que lorsqu'elle est associée à d'autres carences en vitamines du groupe $\mathrm{B}$. Le bilan de

TABLEAU 7 B

Bilans vitaminiques

(Expérience B)

Nombre d'animaux par lot : 7

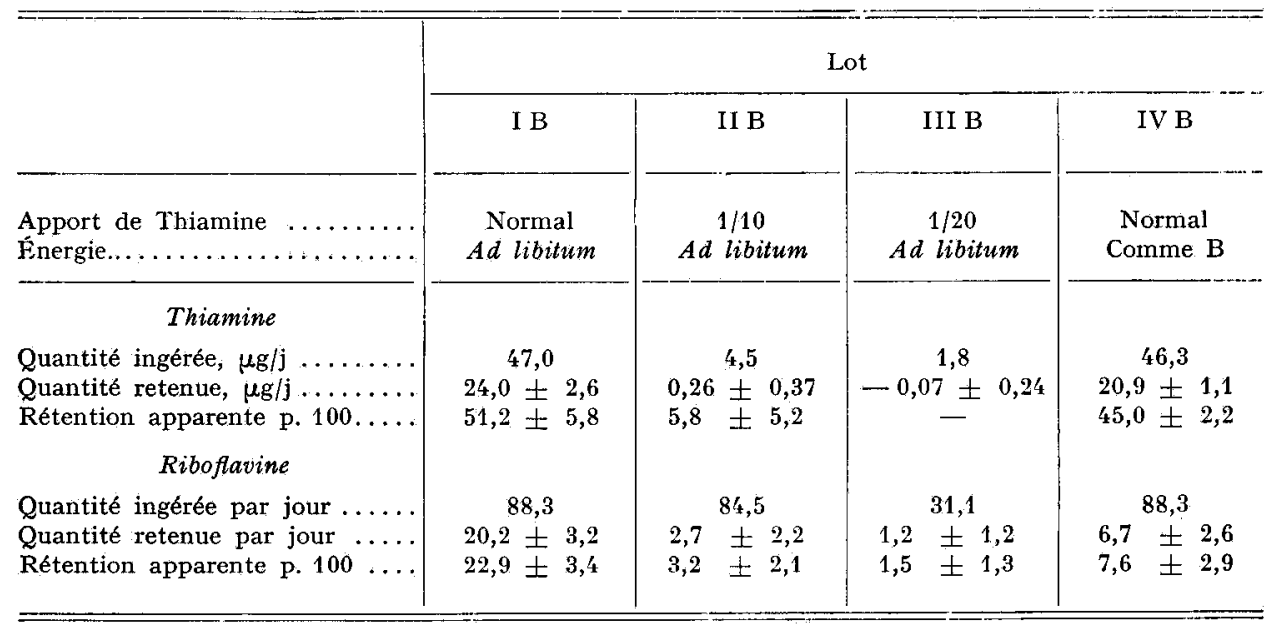

thiamine diminue très fortement à mesure que la dose administrée décroît, pour devenir négatif à la dose de $\mathrm{I}, 8 \mu \mathrm{g} / \mathrm{j}$; contrairement à l'expérience $\mathrm{A}$, il en va de même de la rétention exprimée en valeur relative. Il est intéressant de noter par ailleurs que les animaux du lot IV B, qui ont reçu la même dose de thiamine que ceux du lot $I$, 
ont stocké pratiquement la même quantité de cette vitamine dans leurs tissus, malgré une restriction énergétique de $37 \mathrm{p}$. Ioo. On peut en conclure qu'au-dessus d'une certaine dose journalière, le dépôt de thiamine dans les tissus, et plus particulièrement dans le foie, est directement proportionnel à la quantité ingérée, tout au moins dans nos conditions expérimentales.

La diminution de la rétention de thiamine, sous l'effet d'une carence plus ou moins sévère en cette vitamine, est accompagnée dans tous les cas d'une réduction du dépôt de riboflavine ; cette réduction est la conséquence directe de la dépression de croissance provoquée par la carence en thiamine, en raison de la constance de la concentration par rapport au poids sec.

$3^{\circ}$ Une carence en riboflavine seule (tab1. $7 \mathrm{C}$ ) se traduit par une diminution très notable du dépôt de cette vitamine, tandis que le coefficient de rétention est fortement

\section{TABLEAU $7 \mathrm{C}$}

Bilans vitaminiques

(Expérience C)

Nombre d'animaux par lot : 7

\begin{tabular}{|c|c|c|c|}
\hline & \multicolumn{3}{|c|}{ Lot } \\
\hline & I C & II C & III C \\
\hline Apport de riboflavine ...... & Normal & $1 / 10$ & $1 / 20$ \\
\hline Thiamine & & & \\
\hline Quantité ingérée, $\mu \mathrm{g} / \mathrm{j} \ldots \ldots \ldots \ldots \ldots \ldots \ldots$ & 47,96 & 47,92 & 47,55 \\
\hline Quantité retenue, $\mu g / j \ldots \ldots \ldots \ldots \ldots \ldots$ & $12,34 \pm 0,88$ & $11,59 \pm 0,11$ & $10,23 \pm 0,86$ \\
\hline Rétention apparente p. $100 \ldots \ldots \ldots \ldots$ & $25,73 \pm 1,82$ & $24,20 \pm 2,25$ & $21,55 \pm 1,89$ \\
\hline Riboflavine & & & \\
\hline Quantité ingérée, $\mu \mathrm{g} / \mathrm{j} \ldots \ldots \ldots \ldots \ldots$ & 89,9 & 8,97 & 4,46 \\
\hline Quantité retenue, $\mu \mathrm{g} / \mathbf{j} \ldots \ldots \ldots \ldots \ldots \ldots$ & $24,44 \pm 0,98$ & $8,81 \pm 0,84$ & $5,71 \pm 0,58$ \\
\hline Rétention apparente p. $100 \ldots$ & $27,2 \pm 1,03$ & - & - \\
\hline
\end{tabular}

accru et dépasse même roo $\mathrm{p}$. Ioo. Il faut noter cependant que le fait de négliger la contribution de la synthèse microbienne conduit à une surestimation de la rétention en valeur relative.

Contrairement à ce que l'on observe pour la riboflavine, le dépôt de thiamine dans les tissus n'est pas affecté par une carence en riboflavine seule. Il est indépendant de la dépression de croissance provoquée par la carence en riboflavine et dépend surtout de la quantité de thiamine ingérée. A l'inverse, le dépôt de riboflavine est en relation étroite avec l'intensité de croissance permise par le régime et non avec la quantité ingérée. La figure 4 illustre d'une façon parfaite le contraste entre ces deux vitamines. 


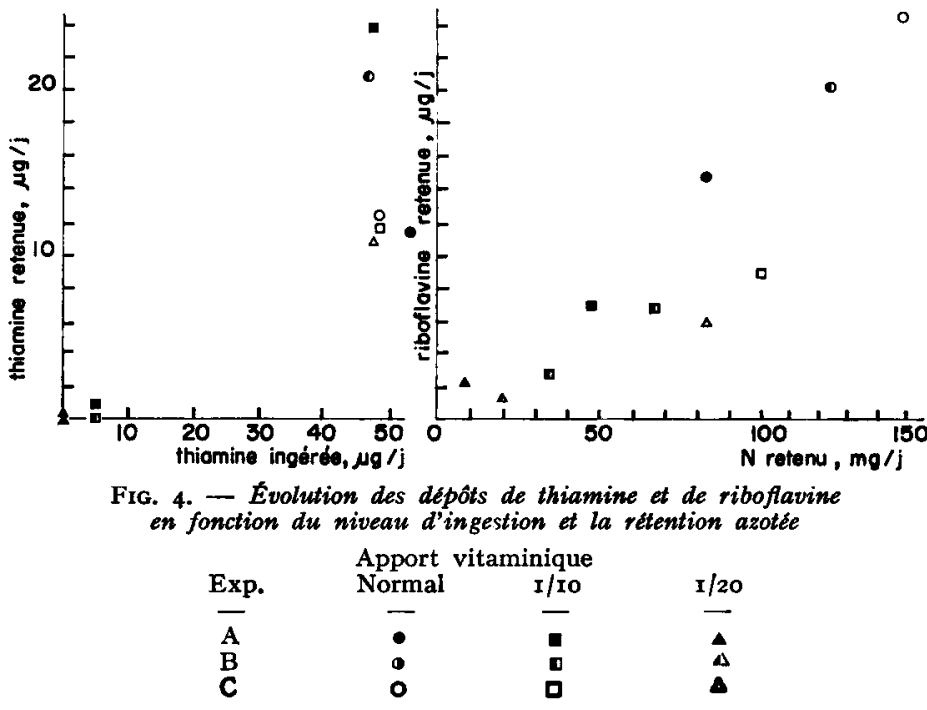

\section{DISCUSSION}

Il est maintenant établi que la consommation spontanée d'énergie chez le Rat, dans les conditions de l'alimentation séparée, est en relation directe avec l'intensité de la protéinogénèse, elle-même fonction de l'apport azoté quantitatif et qualitatif (HENRY et RÉRAT, I966). Les résultats de la présente étude ont montré que cet ajustement de la consommation d'énergie, en fonction de la protéinogénèse, est également retrouvé lors de modifications plus ou moins importantes de l'apport vitaminique, qu'il s'agisse de l'ensemble des vitamines B ou simplement de la thiamine ou de la riboflavine. On peut dès lors se demander si l'action d'une carence vitaminique sur l'appétit intervient d'une manière spécifique ou plus généralement par l'intermédiaire de la rétention d'azote dans les tissus.

On connaît en effet le rôle considérable des vitamines du groupe B dans les mécanismes de synthèse des protéines, d'après les nombreuses revues consacrées à ce problème (MitchelL, I943 ; Terroine, I952 ; Jacquot et Adrian, I964 ; TERRoINe, 1966). Une carence en thiamine exalte le catabolisme azoté (JACQuOr et THrvoli.E, 1941 ; BINET et WELLERS, I964 ; WELLERS et al., I964) ; une ariboflavinose provoque chez le Rat une hypoprotéinémie et une déviation du métabolisme de certains acides aminés, dont le tryptophane (GAUDIN-Harding et al., I964). En d'autres termes, 1a fraction azotée du régime n'est que partiellement utilisable à la suite d'une carence plus ou moins sévère en vitamines.

Nous devons conclure que, même dans le cas d'une carence en vitamines, la consommation spontanée d'énergie demeure réglée par le niveau des synthèses protidiques. La dépression de l'appétit provoquée par une avitaminose ne constituerait pas un effet spécifique de telle ou telle vitamine, mais résulterait d'un équilibre spontané entre la consommation et le niveau du facteur limitant de la croissance, et plus 
précisément de la protéinogénèse (dans le cas présent de l'apport vitaminique) Comme 1'a d'ailleurs fait remarquer LE MAGNEN (I95I), ce phénomène d'inappétence dans les avitaminoses s'expliquerait par le fait que 1'aliment a perdu ses propriétés nutritionnelles par défaut d'utilisation métabolique.

En dehors de son action sur le niveau d'ingestion des nutriments énergétiques, l'avitaminose B est susceptible de modifier plus ou moins profondément l'utilisation métabolique de ces derniers (BLAXTER, I964). La comparaison des lots carencés ou non en thiamine, à niveau énergétique égal, dans l'expérience $\mathrm{B}$, confirme le rôle particulier de cette vitamine dans 1'anabolisme des graisses à partir des glucides (McHENRY et CoRneTt, I944). Utilisant la méthode paired feeding, Voris et al. (I942 $a, b$ ) ont mis en évidence l'influence favorable d'une supplémentation en thiamine et en riboflavine sur le dépôt de lipides chez le Rat en croissance, indépendamment de l'action sur l'appétit. De leur côté, Lockrart et al. (rg66) constatent qu'un apport insuffisant de thiamine entraîne chez le Poulet une diminution sensible de la valeur énergétique du régime, exprimée en énergie métabolisable, alors que les autres vitamines du groupe B, en particulier la riboflavine, sont sans influence notable. L'importance considérable des vitamines du groupe $B$ dans l'utilisation globale de l'énergie est d'ailleurs prévisible, compte tenu de leur intervention au niveau des processus enzymatiques du métabolisme intermédiaire (TERROINE, I964 ; STIEALU et al., I965).

Dans l'établissement des bilans vitaminiques, nous avons été amenés à négliger la contribution de la microflore intestinale dans la synthèse vitaminique, de même que les processus d'absorption, d'élimination et de dégradation. Or, il a été montré que la synthèse de vitamines par la microflore varie dans des limites assez considérables, selon le type de régime, et plus particulièrement selon la nature des glucides (RÉRAT, I964; HOTZEL, I966). Lorsque la fraction énergétique du régime est constituée par du saccharose (ce qui est le cas dans la présente étude), la synthèse de thiamine par la microflore est de toute façon réduite ; ceci est confirmé par la diminution de la concentration du contenu intestinal en thiamine, à mesure que la carence s'accentue. En ce qui concerne la riboflavine, par contre, l'interprétation apparaît obscure. Si l'on se réfère aux résultats des expériences $A$ et $C$, on constate effectivement qu'une carence en cette vitamine aboutit à une augmentation très sensible de la rétention en valeur relative, si l'on ne tient compte que de la quantité ingérée. Nous pouvons dès lors tenter de conclure à une élévation de la synthèse microbienne consécutive à la carence. A cela, une explication peut être donnée : les travaux de GERSHOFF et al. (r959), sur le Chat, sembleraient indiquer une action favorable du saccharose sur la synthèse de riboflavine par la microflore intestinale, malgré 1'avis contraire de la plupart des auteurs. On peut se demander s'il n'en serait pas de même dans nos conditions expérimentales. Il convient toutefois de noter que la microflore intestinale n'est pas le seul facteur responsable de l'augmentation apparente du coefficient de rétention vitaminique. Cette augmentation pourrait être due, en particulier, à une élimination réduite dans le cas d'une déficience, la riboflavine n'étant libérée qu'à partir des flavoprotéines en fonction des besoins (BLUM, I966). Quoi qu'il en soit, quel que soit le mécanisme en cause, nous devons reconnaître qu'une carence en riboflavine produit un effet moins brutal, sur le niveau d'ingestion, qu'une carence en thiamine.

La comparaison des quantités de vitamines stockées dans l'organisme a fait apparaître une différence fondamentale dans les effets réciproques de la thiamine et 
de la riboflavine. Ainsi, à la suite d'une carence en thiamine, on observe un épuisement hépatique presque complet et très rapide en cette vitamine (TERROINE, I957), accompagné d'une diminution du dépôt de riboflavine, alors qu'une carence en cette dernière (SURE et Ford, I942 ; Sure, I945 ; Singher et al., I944; Bhagvat et Devi, I949) n'exerce aucun effet dépressif sur la quantité de thiamine fixée par les tissus. A la lumière de nos résultats, nous devons cependant interpréter avec prudence la relation de proportionnalité entre le dépôt apparent de thiamine et la quantité ingérée. Cette relation n'implique pas qu'aux doses indiquées il y ait nécessairement une mise en réserve de thiamine dans les tissus, étant donné que cette dernière est associée aux enzymes actifs ; il est possible, au contraire, qu'à mesure que s'accroît la rétention de thiamine, son élimination augmente dans les mêmes proportions et reflète ainsi l'activité métabolique de 1'organisme. Dans le cas de la riboflavine, par contre, les variations relativement faibles de sa teneur dans l'organisme sous l'effet d'une carence et la relation de proportionnalité entre son dépôt et l'intensité de croissance démontrent une fois de plus, à la suite des travaux de BLUM (I966), la solidarité étroite entre cette vitamine et les protéines labiles du sérum qui sont susceptibles de la complexer à l'état de flavoprotéines.

En définitive, l'administration au Rat de régimes plus ou moins déficients en vitamines $B$, ou simplement carencés en thiamine ou en riboflavine, nous a permis de montrer que la consommation spontanée d'énergie, dans les conditions de l'alimentation séparée, est déterminée pour une large part par l'intensité de la protéinogénèse, elle-même fonction de la sévérité de la carence vitaminique selon des modalités particulières à chacune des vitamines. Ceci semble exclure une intervention directe des vitamines sur 1'appétit ; ces dernières agiraient seulement indirectement en règlant le métabolisme. Il s'agit là d'un phénomène général d'ajustement de la consommation d'énergie en fonction du niveau du facteur limitant de la protéinogénèse.

Reçu pour publication en juillet 1969.

\section{SUMMARY}

EFFECT OF SUPPLY OF VITAMINS B ON VOLUNTARY INTAKE OF ENERGY BY GROWING WHITE RATS

Three experiments (A, B and C) were conducted with 155 male Wistar CF rats after weaning, of initial weights from 50 to $70 \mathrm{~g}$, to study the effects of more or less severe multiple deficiency of vitamins of the B complex (Exp. A) or simple deficiency of thiamine (Exp. B) or of riboflavine (Exp. C) on voluntary intake of energy and metabolic utilization of nitrogenous and energysupplying nutrients. After they had been depleted of vitamins the rats were fed on the method of separate feeds. For this they were given in two separate feed boxes :

- a protein diet supplying a fixed amount of protein daily in the form of devitaminized casein supplemented with I.2 g cystine in the exp. A and I.89 in exp. B and C, and three different amounts of vitamins, a dose corresponding to the usual recommendations (group I), one tenth of that amount (group II) or one twentieth (group III) ;

- a protein-free diet based on saccharose offered to appetite.

In exp. B there was a fourth group (IV) in which the rats got the normal amount of vitamins and the same amount of energy as those in group II (restricted to one tenth).

At the end of the experiment, which lasted 28 days (Exp. A and C) or $I_{4}$ days (Exp. B) dry matter, ash and nitrogen were estimated in carcasses after removal of the intestines and their 
contents (Exp. A) and of livers (in all three experiments) and thiamine and riboflavine were estimated microbiologically in these three fractions.

More or less severe deficiency of vitamins $B$ reduced growth rate, more when the deficiency was more severe (tables $3 \mathrm{~A}, 3 \mathrm{~B}, 3 \mathrm{C}$ ). This depression of growth was accompanied by reduction of voluntary intake of energy, but this latter (EI) was in every case directly proportional to the amount of nitrogen fixed in the tissues according to the equation (fig. 3 ) :

EI $(\mathrm{kcal} /$ day $)=18.74+0.309 \mathrm{~N}$ (mg/day), with a correlation coefficient $r=0.976$

Changes in vitamin contents in intestine, liver and carcass (tables $6 \mathrm{~A}, 6 \mathrm{~B}, 6 \mathrm{C}$ ) showed, mainly in liver, a direct relation between deposition of thiamine in the tissues and the amount of this vitamin ingested, in the conditions of the experiment. There were, respectively, 375-43 and $3^{8} \mu \mathrm{g}$ in the whole organism for intakes of $47.0-4,5$ and $\mathrm{r} .8 \mu \mathrm{g}$ per day. The amounts of riboflavine per $g$ dry weight were little modified by a deficiency of this vitamin, although the deposition of riboflavine increased in proportion to gain in weight and more closely to retention of nitrogen (fig. 4).

The results can be taken to exclude a direct effect of vitamins on appetite. They act only indirectly in regulating metabolism according to the particular mode of action of each one of these vitamins. The effect here is a general adjustment of intake of energy in relation to the degree to which protein formation is limited.

\section{RÉFÉRENCES BIBLIOGRAPHIQUES}

Atwater W. O., Bryant A. P., 1903. The chemical composition of american food materials. $U . S$. Dept Agr. Bull., 28.

Bhagvat K., Devi P., r949. Interrelationship of certain vitamins of the B group in aneurin, riboflavin and biotin deficiencies. Biochem. $J ., 45,32-38$.

Binet L., Wellers G., ig64. Contribution à l'étude du métabolisme de l'azote. Rôle de la vitamine $B_{1}$ (thiamine). C. R. Acad. Sci. Paris, 258, I3I5-1317.

Blaxter K. L., I964. Dietary factors affecting energy utilization. Proc. Nutr. Soc., 23, 3-Ir.

Blum J. C., I966. Contribution à l'étude du métabolisme de la riboflavine au cours de l'ovogénèse de Gallus Gallus $L$. Thèse Fac. Sci. Univ. Paris, 162 p.

Gaudin-Harding F., Jacquot-Armand Y., Boffa G. A., Blum J. C., I965. Influence de la carence en riboflavine sur les protéines sériques du Rat blanc. Ann. Nutr. Alim., 19, C 633-544.

Gershoff S. N., Anprus S. B., Hegsted D. M., I959. The effect of the carbohydrate and fat content of the diet upon the riboflavine requirement of the cat. $J$. Nutr., 68, 75-88.

HENRY Y., RERAT A., I962. Influence de la qualité et de la quantité des matières azotées ingérées sur la consommation spontanée d'énergie chez le Rat en croissance. Ann. Biol. anim. Bioch. Biophys., 2 , $267-276$.

HenRY Y., RÉRAT A., I964. Influence du niveau d'ingestion des vitamines B sur la consommation spontanée d'énergie chez le Rat en croissance. C. R. Acad. Sci., 258, 3915-3918.

HenRy Y., Rérat A., Ig66. Évolution de l'ingestion spontanée de principes énergétiques en fonction de la vitssse de croissance et de la protéinogénèse chez le Rat blanc. Cahiers AEC, $\mathrm{n}^{\circ} 6$, Aminoacides, $^{\circ}$ protides, protéines, 239-262, AEC, Paris.

Hötzel D., rg66. Contributions of the intestinal microflore to the nutrition of the host. Vitam. Horm., 24, II5-I 7 I.

JACquot R., Adrian J., I964. Le besoin quantitatif des diverses vitamines en fonction des constituants non vitaminiques du régime. Ann. Nutr. Alim., 18, $\mathrm{C}_{4 \mathrm{I}-86 .}$

Jacouot R., Thivolle L., I94I. Métabolisme de l'azote et vitamine $B_{1}$. C. R. Soc. Biol., 135, I403. I405.

Le Magnen J., r95r. Quelques aspects des liens entre sensibilité chimique et appétit. Ann. Nutr. Alim., 5, 393-409.

Lockhart W. C., Bryant R. L., Bolin D. W., I966. The effects of B vitamin deficiencies on the efficiency of metabolizable energy and protein utilization. Poultry Sci., 45, 939-945.

Mc Henry E. W., Cornetr M. L., 1944. The role of vitamins in the anabolism of fats. Vitam. Horm., 2, I-27.

Mitchell H. H., r943. The chemical and physiological relationship between vitamins and aminoacids. Vitam. Horm., 1, 157-194.

Morgan T. B., YUdKin J., 1962. The vitamin-sparing action of sorbitol, sugars and related substances. Vitam. Horm., 20, 39-66. 
RGRAT A., I960. Synthèse et absorption des vitamines $B$ dans le tube digestif du mouton adulte. Thèse Fac. Sci. Univ. Paris, II 2 p., I. N. R. A., Paris.

RÉrat A., I964. Rôle de la flore digestive dans la production des vitamines chez les monogastriques et chez les polygastriques. Ann. nutr. alim., 13, C $187-\mathrm{C} 265$.

RÉrat A., HENRY Y., r963. Étude de l'ajustement de la consommation spontanée d'énergie en fonction de l'apport azoté chez le rat en croissance. Ann. Biol. anim. Bioch. Biophys., 3, 263-298.

Rérat A., Henry Y., I966. Relations entre la consommation énergétique et la nature du régime.

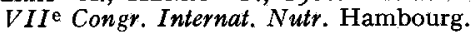

Rérat A., Henry Y., Jacquot R., I963. Relation entre la consommation spontanée d'énergie et la rétention azotée chez le Rat en croissance. C. R. Acad. Sci. Paris, 256, 787-789.

SARETT H. P., Cheldelin Y. H., r944. The use of Lactobacillus fermentum 36 for thiamine assay. J. Biol. Chem., 145, 153-I6o.

Singher H. O., Kensler C. J., Levy H., Poore E., Rhoads C. P., Unna K., I944. J. Biol. Chem., 154, 69.

Snell E. E., Strong F. M., I949. A microbiological assay for riboflavin. Indust. Eng. Chem. Ann. Ed., $11,346 \cdot 350$.

Stiealu W. J., Freedland R. A., Meyer J. H., 965. Effects of B Vitamin deficiencies and of starvation on liver enzyme activities of growing rats. J. Nutr., $\mathbf{7 8}$, ro9-1 6 .

SurE B., FORD Z. W., 1942. Vitamins interrelationship. Thiamin and riboflavin interrelationships in metabolism. J. Biol. Chem., 146, 24. I-250.

Terroine E. F., I952. La synthèse protéique, 539 p., C. N. R. S., Paris.

TERroine E. F., VALla S., 1933. Valeur comparée des différents aliments protéiques pour la croissance. C. R. Acad. Sci. Paris, 108, 288-290.

Terroine T., I957. Les caractères du rôle vicariant de l'acide ascorbique en avitaminose $\mathrm{B}_{\mathbf{1}}$. Arch. Sci. Physiol., 11, 273-301.

Terroine T., r964. Le contrôle vitaminique des activités enzymatiques. Ann. Nutr. Alim., 19, C 633644.

Terrorne T., I966. Les interyelations vitaminiques, 272 p., C. N. R. S., Paris.

Voris L., BLACK A., Swift $\quad$ R. W., Frencr $\quad$ C. E., 1942. Thiamine, riboflavine, pyridoxine and pantothenate deficiencies as affecting the appetite and growth of the albino rats. J. Nutr., 23, 555-566.

Voris L., Moore H. P., I942. Thiamina, riboflavin, pyridoxine and pantothenate deficiencies as affecting the body composition of the albino rat. J. Nutr., 21, 7-г6.

Wellers G., Leluan M., Chevan J., I954. Re herches sur le métabolisme du soufre. I2. Influence de la carence en vitamine $B_{1}$ sur 13 mśtabolisme du soufre et də l'azote chez le rat blanc adulte. Arch. Sci. Physiol, 18, 265-275. 